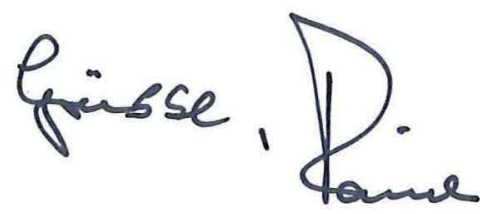

\section{CARBON 13 IN PACIFIC DEEP AND INTERMEDIATE WATERS, 0-370 KA: IMPLICATIONS FOR OCEAN CIRCULATION AND PLEISTOCENE $\mathrm{CO}_{2}$}

\author{
A.C. Mix, N.G. Pisias, R. Zahn,1 W. Rugh, C. \\ Lopez, and K. Nelson² \\ College of Oceanography, Oregon State University, \\ Corvallis
}

depth gradient favors a North Atlantic origin of the phenomenon, although local variations of Pacific intermediate water masses can not be excluded at present.

\section{INTRODUCTION}

The ice core record of Pleistocene atmospheric $\mathrm{C}^{\prime} \mathrm{O}_{2}$ variations [e.g., Barnola et al., 1987] challenges paleoceanographers to find mechanisms controlling atmospheric $\mathrm{pCO}_{2}$ by ocean circulation. The ocean is a logical place to look for $\mathrm{CO}_{2}$ controls. It holds the largest easily exchangeable carbon reservoir on Earth, $>50$ times the carbon resident in the atmosphere [Broecker et al., 1980]. Stable carbon isotope data from benthic and planktic foraminifera [Shackleton and Pisias, 1985] suggest that the distribution of carbon within the ocean controls atmospheric $\mathrm{pCO}_{2}$. The mechanism remains unclear, however.

Broecker [1982] proposed that variations in oceanic carbon storage responded to nutrient content of the ocean. In this scenario, erosion of continental shelves during low sea level stands resulted in high oceanic nutrients during glacial time. Higher nutrients would drive more productivity, and a greater fraction of upwelled carbon would be fixed as organic matter. This would pump more carbon into the deep sea as falling detritus. Two lines of evidence reject this model as a source of $\mathrm{pCO}_{2}$ change. The ocean's carbon isotopic changes occurred earlier than the shelf erosion model would predict [Shackleton and Pisias, 1985]. Cadmium/ calcium content in benthic foraminifera, a nutrient proxy, suggest that the ocean's nutrient budget has not changed much [Boyle and Keigwin, 1985,1986]. 
Thus, the search for mechanisms shifted to examining variations in ocean circulation.

Several ocean circulation scenarios have been suggested that could explain changes in atmospheric $\mathrm{pCO}_{2}$ without significantly changing the global oceanic nutrient budget. Most focus on the Antarctic, because here high nutrients and $\mathrm{pCO}_{2}$ are present in surface waters and a relatively direct link into the deep sea exists. These mechanisms change the efficiency of the Antarctic biota, or the interaction of Antarctic surface waters with cold deep and/or warm surface waters [Knox and McElroy, 1985; Siegenthaler and Wenk, 1984; Sarmiento and Toggweiler, 1985; Keir, 1988; Lyle and Pisias, 1990]. Referred to as the "polar nutrient" hypotheses, all require radical decreases in surface ocean nutrients in the southern ocean at glacial maxima. Data from the Antarctic are not consistent with this prediction. Carbon isotope data from planktonic foraminifera argue that Antarctic surface water nutrients increased at the last glacial maximum [Labeyrie and Duplessy, 1985], while Cd/Ca data suggest little or no change [Boyle, 1988]. Also, most of the polar nutrient hypotheses predict very low deep-sea oxygen, approaching anoxia in glacial time. This, too, has not been observed [Pedersen et al., 1988].

\section{IMPORTANCE OF INTERMEDIATE WATERS}

To avoid the problems of the polar nutrient models, Boyle [1986] posed another ice age scenario, in which the mid-depth ocean was ventilated more than at present by intermediate water formation and less by deepwater formation. Boyle [1986] argued that doubling overturn of intermediate waters would increase nutrient fluxes into surface waters and thus increase productivity. Although much of the falling organic matter would be recycled, a fraction would "leak" into the deep sea, where most of it would decay to $\mathrm{SCO}_{2}$. Because overturn in the deep sea is slow, the released carbon would reside longer here than in intermediate waters. The net result is a transfer of carbon and nutrients from the upper ocean to the deep sea, thus reducing the $\mathrm{pCO}_{2}$ in the surface ocean and atmosphere. We will call this redistribution of nutrients and carbon the "Boyle effect".

Boyle [1988] added an alkalinity amplifier to the nutrient transfer scenario. In several scenarios considered, higher input of metabolic $\mathrm{CO}_{2}$ to deep waters lowers deep-sea carbonate ion content. This temporarily increases carbonate dissolution. Alkalinity then increases slowly, until the carbonate ion concentration returns to its steady state value. This process requires a few thousand years, with a time constant which depends on the rates of river input of carbonate and deep-sea dissolution. The alkalinity increase converts a portion of the ocean's dissolved $\mathrm{CO}_{2}$ into carbonate and bicarbonate, thereby reducing atmospheric $\mathrm{pCO}_{2}$.
The exact mechanism by which nutrients and carbon are redistributed is not yet defined. For example, Keir [1988] argued that enhanced Antarctic productivity would move nutrients from intermediate to deep waters as well as Boyle's [1986] intermediate water flushing scenario. Boyle [1988] also noted that increased flushing of intermediate water is by itself not sufficient to reduce atmospheric $\mathrm{CO}_{2}$. The result is highly dependent on the depth of regeneration of organic matter into dissolved $\mathrm{CO}_{2}$, carbonate, and bicarbonate. The transport of organic matter from the intermediate to deep boxes in this model is $10 \%$ of the particulate flux leaving the surface. Lyle and Pisias [1990] argue from sediment trap studies that an export value of about $2 \%$ would be more appropriate [Suess, 1980]. Boyle [personal communication, 1990] disagrees, and notes that the $10 \%$ value is needed for the model to simulate modern nutrient distributions. If $10 \%$ export of carbon below $2500 \mathrm{~m}$ is too high, then the model overestimates the sensitivity of depth transfers of nutrients and carbon to circulation change. In other ways, however, the box model may underestimate the true sensitivity by averaging many local effects into a few boxes. This is an inherent limit to box model studies. Boyle correctly treats his studies as sensitivity tests of viable hypotheses, rather than as true simulations of the real ocean.

All of Boyle's [1988] ice age scenarios predict higher $\delta 13 \mathrm{C}$ gradients between global average intermediate and deep waters. Cofer-Shabica and Peterson [1986], Boyle and Keigwin [1987], Oppo and Fairbanks [1987, 1990], and Zahn et al. [1987] all infer significant ice age nutrient depletion of Atlantic intermediate waters at the last glacial maximum from $\delta^{13} \mathrm{C}$ in Caribbean and North Atlantic benthic foraminifera. Their data suggest large intermediate-to-deep water ${ }^{13} \mathrm{C}$ gradients in the North Atlantic, ranging from about $-0.2 \% o$ at present to $+0.7 \%$ at the last glacial maximum. Compilations of North Atlantic $\delta 13 \mathrm{C}$ data from glacial maxima at $\sim 18 \mathrm{ka}$ [Duplessy et al., 1988] and $\sim 135 \mathrm{ka}$ [Duplessy and Shackleton, 1985] give average intermediate-to-deep water $\delta{ }^{13} \mathrm{C}$ gradients of 0.5 to $0.7 \%$, with gradients as large as $1.0 \%$ in the lowlatitude North Atlantic. Kallel et al. [1988] find a similar pattern in carbon isotope data from the northeastern Indian Ocean and the Arabian Sea. They infer a sharp hydrographic front near $\sim 2 \mathrm{~km}$ depth at the last glacial maximum. Above this depth, waters were enriched in ${ }^{13} \mathrm{C}$ by $0.5 \%$ (Arabian Sea) to $1.0 \%$ (Bay of Bengal) relative to deep waters in the same area. Zahn and Pedersen [1990], however, suggest that this feature in the Indian Ocean at the last glacial maximum may not be repeated during earlier glacial events.

Cadmium/calcium content of benthic foraminifera from North Atlantic sites show an analogous pattern to that of $\delta^{13} \mathrm{C}$ [Boyle and Keigwin, 1987]. Today the intermediate-to-deep water gradient here is essentially zero. At the last glacial maximum, deepwater sites $(>2.5 \mathrm{~km}$ ) have higher $\mathrm{Cd} / \mathrm{Ca}$ (higher 
nutrients) than at present, and the intermediate-todeep water $\mathrm{Cd} / \mathrm{Ca}$ gradient was about $0.12 \mu \mathrm{mol}$ $\mathrm{mol}^{-1}$. This would be equivalent to a phosphate gradient of about $1.4 \mu \mathrm{mol} \mathrm{kg-1}$, or a $\delta 13 \mathrm{C}$ gradient of about $1.1 \%$.

If the Boyle effect is to work quantitatively as a $\mathrm{CO}_{2}$ mechanism, intermediate-to-deep water $\delta^{13} \mathrm{C}$ gradients must be very large at the glacial maximum. Boyle's [1988] box model results scale such that an increase in this gradient by $1 \%$ would accompany a 20-60 ppm decrease in atmospheric $\mathrm{pCO}_{2}$ (as compared to a total ice age $\mathrm{pCO}_{2}$ change of $\sim 90 \mathrm{ppm}$ observed in ice cores). Note that this value applies to the global average $\delta{ }^{13} \mathrm{C}$ gradient, not a local or regional extreme. Data from the Pacific are needed to evaluate the global $\delta^{13} \mathrm{C}$ budget in intermediate waters. So far, evidence for lower nutrients in Pacific intermediate waters during glacial time is equivocal. Carbon isotope data from the last glacial maximum in the Pacific [Duplessy et al., 1988] allow an intermediate-to-deep water $\delta^{13} \mathrm{C}$ gradient of as much as $0.5 \%$, but scatter in the data precludes a constrained interpretation. Cadmium/calcium data in benthic foraminifera from a 1600 -m deep site in the Sea of Okhotsk suggest ice age values $0.03 \pm 0.04$ $\mu \mathrm{mol} \mathrm{mol}^{-1}$ lower than at present [Boyle, 1988]. This would be equivalent to phosphate concentration decrease of $\sim 0.4 \mu \mathrm{mol} \mathrm{kg-1}$, or a $\delta^{13} \mathrm{C}$ increase of $\sim 0.3 \%$. Again, the error bars make detailed interpretation difficult. To what extent the ice age ${ }^{13} \mathrm{C}$ enrichment of intermediate relative to deep waters is a consistent feature of all glacial maxima and to what extent it is a global effect remain an open question. To constrain the interpretations further, we provide data on carbon isotope gradients between intermediate and deep-water sites in the eastern tropical Pacific and extend the time series considered to $\sim 370 \mathrm{kyr}$.

\section{RESULTS AND DISCUSSION}

\section{Data and Age Models}

New data are presented from two sites in the eastern equatorial Pacific; core RC13-110 from $0.10^{\circ} \mathrm{N}, 95.65^{\circ} \mathrm{W}$, at $3231 \mathrm{~m}$ water depth, and core V19-27, from $0.47^{\circ} \mathrm{S}, 82.07^{\circ} \mathrm{W}$, at $1373 \mathrm{~m}$ water depth. In addition, we compare our data to the widely used benthic foraminiferal stable isotope record from core V19-30 [Shackleton and Pisias, 1985 ], from $3.38^{\circ} \mathrm{S}, 83.52^{\circ} \mathrm{W}, 3091 \mathrm{~m}$ water depth. The three sites are shown in relation to modern water-column $\delta^{13} \mathrm{C}$ in Figure 1.

All analyses in cores RC13-110 and V19-27 were made on a Finnigan/MAT 251 mass spectrometer at Oregon State University. This instrument is equipped with an Autoprep Systems automated carbonate device. Precision of a local carbonate standard over a 1-year period (1989) is $\pm 0.09 \%$ o for $\delta 18 \mathrm{O}$, and $\pm 0.04 \%$ o for $\delta^{13} \mathrm{C}( \pm 1 \sigma$, $\mathrm{n}=229$ ). For the primary calibration standard (NBS

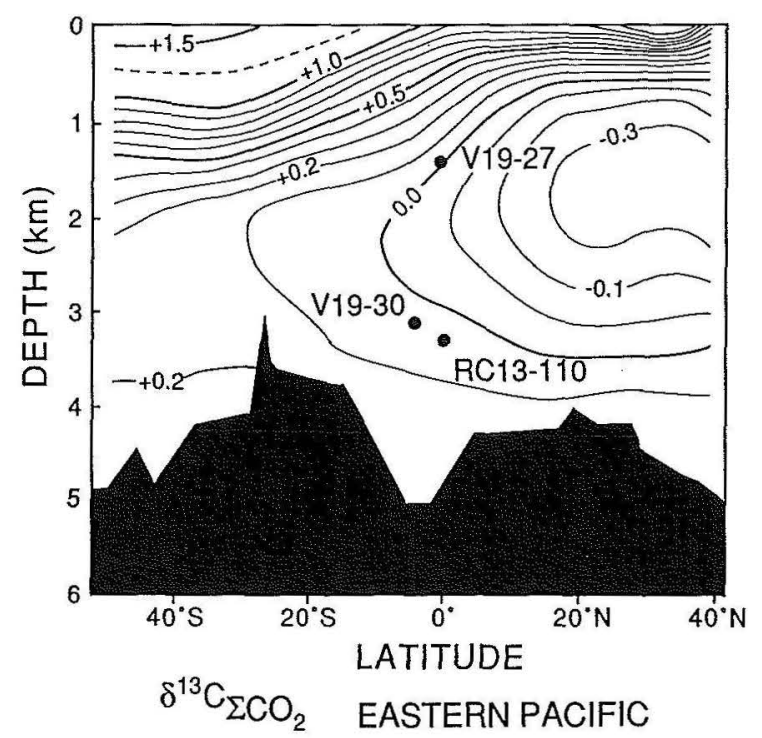

Fig. 1. Locations of cores V19-27, V19-30, and RC13-110, plotted on a cross section of water column $\delta 13 \mathrm{C}$ in the eastern Pacific (contours after Kroopnick [1985]).

20 ), precision was $\pm 0.04 \%$ for $\delta 18 \mathrm{O}$, and $\pm 0.03 \%$ o for $\delta{ }^{13} \mathrm{C}( \pm 1 \sigma, \mathrm{n}=71)$. These standards were measured at the same time, and over the same size range, as the samples and thus should reflect the analytical precision associated with mass spectrometry reasonably well. The precision of foraminiferal analyses is somewhat worse than this, due to true variability within the foraminifera, benthic mixing, and sample sizes which limit a true statistical sampling of the mean population. Based on 48 replicate analyses of benthic foraminifera in V19-27, we estimate the precision $( \pm 1$ standard deviation) of foraminiferal analyses to be $\pm 0.15 \%$ for $\delta 18 \mathrm{O}$, and $\pm 0.08 \%$ for $\delta^{13} \mathrm{C}$. We use these values to estimate the noise level of individual analyses throughout our data set.

Following normal practice, all data are expressed versus the international Pee Dee Belemnite (PDB) scale. In core V19-27 all analyses are from the species Cibicides wuellerstorfi. In core RC13-110, most of the analyses are from the $C$. wuellerstorfi, although some are from the genus Uvigerina. The isotope data are plotted in Figure 2, with $\delta 18 \mathrm{O}$ in Cibicides corrected by $+0.64 \%$ [Shackleton, 1974], and $\delta^{13} \mathrm{C}$ in Uvigerina corrected by $+0.90 \%$ o [Duplessy et al., 1984]. All data and our preferred age models are given in Table 1.

Age models in RC13-110 and V19-27 (Figure 3) were generated by correlation to the SPECMAP long time scale [Imbrie et al., 1984], using the method of Martinson et al. [1982]. The age model in V19-30 (Figure 3) is that given by Shackleton and Pisias [1985] but smoothed to eliminate abrupt sedimentation rate changes. Sedimentation rates in 

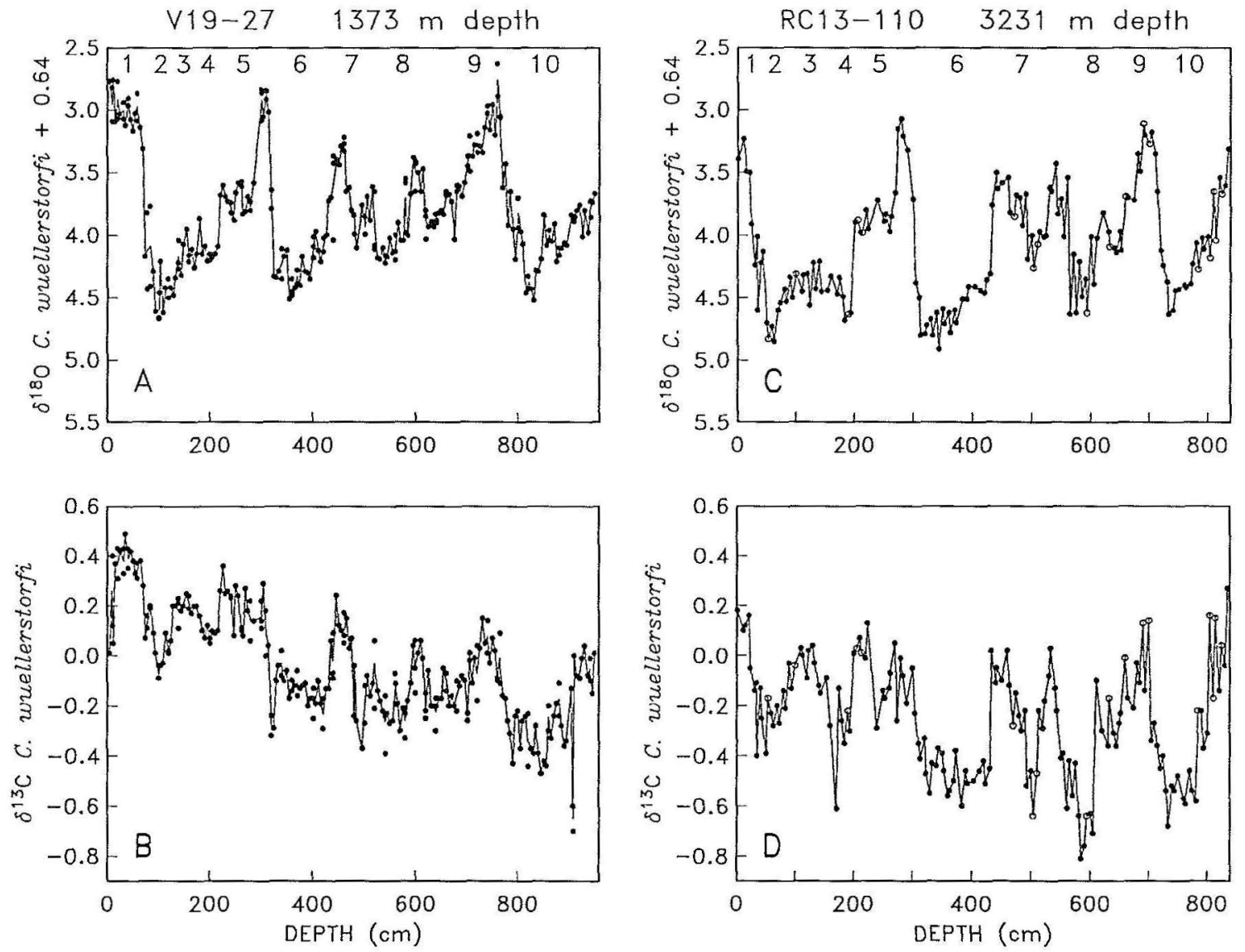

Fig. 2. Stable isotopes of oxygen and carbon in benthic foraminifera, plotted vs. depth in cores V19-27 (from intermediate water depth of $1373 \mathrm{~m}$ ) and RC13-110 (from deep-water depth of 3231 $\mathrm{m})$ in the eastern tropical Pacific. Replicate analyses are shown, but the line passes through the average value at each depth in a core. All analyses in V19-27 are from Cibicides wuellerstorfi: (a) $=\mathrm{V} 19-27 \delta_{18 \mathrm{O}}+0.64,(\mathrm{~b})=\mathrm{V} 19-27 \delta^{13} \mathrm{C}$. In core RC13-110, solid circles indicate Cibicides sp. $(\delta 18 \mathrm{O}+0.64 \%)$, and open circles indicate Uvigerina sp. $(\delta 13 \mathrm{C}+0.90 \%),(\mathrm{c})=\mathrm{RC} 13-110$ composite $\delta 18 \mathrm{O},(\mathrm{d})=\mathrm{RC} 13-110$ composite $\delta^{13} \mathrm{C}$. Traditional $\delta^{18} \mathrm{O}$ stages are labeled, following Shackleton and Opdyke [1973].

these cores average $2.2,2.5$ and $4.8 \mathrm{~cm} / \mathrm{kyr}$, respectively. The average sampling interval is about $2.5 \mathrm{kyr}$ in RC13-110, about $2.0 \mathrm{kyr}$ in V19-27, and about $0.6 \mathrm{kyr}$ in V19-30.

The deep-water record of $\delta 18 \mathrm{O}$ and $\delta^{13} \mathrm{C}$ variations from RC13-110 is similar to the higherresolution record from core V19-30, at $3091 \mathrm{~m}$ water depth [Shackleton and Pisias, 1985] but extends more than twice as far in time. The similarity between these records (Figure 4) is reassuring, because the data in the two cores come from different species. After applying the standard (constant) corrections above, a slight offset remains between $\delta 18 \mathrm{O}$ in RC13-110 and V19-30 (Figure 4a). This may reflect true water mass differences between the sites, but more likely reflects either deviations from the assumed $0.64 \% 0 \delta 18 \mathrm{O}$ offset between Uvigerina and Cibicides, or slight differences in laboratory calibration between the Oregon State University (OSU) and Cambridge University laboratories.

Legitimate concerns have been raised about using Uvigerina $\delta^{13} \mathrm{C}$ data as a proxy for water mass $\delta^{13} \mathrm{C}$ of $\sum \mathrm{CO}_{2}$ as this genus elsewhere has a variable $\delta^{13} \mathrm{C}$ offset from Cibicides [Zahn et al., 1986]. In RC13110 , only 11 of the 139 analyses above $350 \mathrm{kyr}$ are from Uvigerina. As shown in Figure 2d, the corrected Uvigerina $\delta^{13} \mathrm{C}$ data in RC13-110 fit well with the data from Cibicides at depths $<600 \mathrm{~cm}$ $(\sim 270 \mathrm{ka})$. Below this level the fit is not as good, implying that the $+0.9 \%$ correction we applied to $\delta^{13} \mathrm{C}$ in Uvigerina may not be appropriate here. Although we have not analyzed both genera in the same samples in RC13-110, it appears based on adjacent samples that a $\delta^{13} \mathrm{C}$ offset of about $0.7 \%$ 
TABLE 1. Stable Isotope Data and Age Models

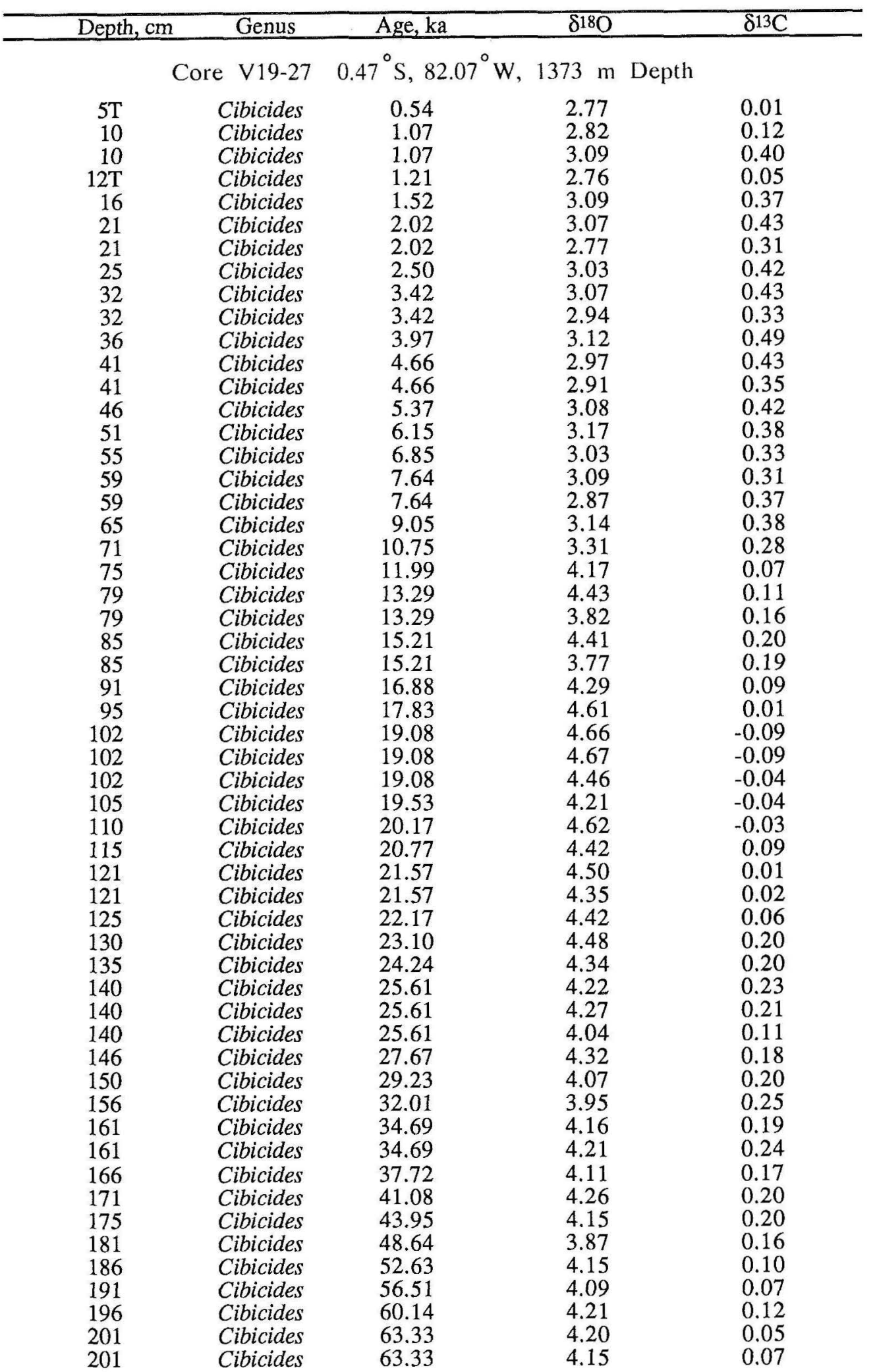


TABLE 1. (continued)

\begin{tabular}{|c|c|c|c|c|}
\hline Depth, cm & Genus & Age, $\mathrm{ka}$ & 8180 & $813 \mathrm{C}$ \\
\hline 205 & Cibicides & 65.61 & 4.18 & 0.10 \\
\hline 210 & Cibicides & 68.07 & 4.15 & 0.09 \\
\hline 216 & Cibicides & 70.79 & 4.09 & 0.10 \\
\hline 221 & Cibicides & 73.16 & 3.68 & 0.26 \\
\hline 226 & Cibicides & 75.85 & 3.60 & 0.36 \\
\hline 231 & Cibicides & 78.94 & 3.69 & 0.25 \\
\hline 235 & Cibicides & 81.66 & 3.73 & 0.26 \\
\hline 242 & Cibicides & 86.84 & 3.82 & 0.23 \\
\hline 242 & Cibicides & 86.84 & 3.74 & 0.24 \\
\hline 247 & Cibicides & 90.52 & 3.88 & 0.08 \\
\hline 251 & Cibicides & 93.36 & 3.66 & 0.28 \\
\hline 256 & Cibicides & 96.63 & 3.58 & 0.24 \\
\hline 262 & Cibicides & 100.10 & 3.57 & 0.11 \\
\hline 262 & Cibicides & 100.10 & 3.61 & 0.10 \\
\hline 265 & Cibicides & 101.70 & 3.83 & 0.08 \\
\hline 270 & Cibicides & 103.97 & 3.81 & 0.27 \\
\hline 275 & Cibicides & 106.03 & 3.69 & 0.18 \\
\hline 280 & Cibicides & 108.09 & 3.80 & 0.22 \\
\hline 280 & Cibicides & 108.09 & 3.73 & 0.06 \\
\hline 286 & Cibicides & 110.95 & 3.58 & 0.14 \\
\hline 301 & Cibicides & 119.53 & 2.84 & 0.22 \\
\hline 301 & Cibicides & 119.53 & 3.09 & 0.11 \\
\hline 301 & Cibicides & 119.53 & 2.87 & 0.14 \\
\hline 305 & Cibicides & 121.72 & 3.06 & 0.29 \\
\hline 310 & Cibicides & 124.16 & 2.92 & 0.00 \\
\hline 310 & Cibicides & 124.16 & 2.85 & 0.18 \\
\hline 315 & Cibicides & 126.44 & 3.02 & 0.04 \\
\hline 321 & Cibicides & 129.00 & 3.64 & -0.24 \\
\hline 321 & Cibicides & 129.00 & 3.79 & -0.32 \\
\hline 326 & Cibicides & 131.11 & 4.33 & -0.29 \\
\hline 330 & Cibicides & 132.81 & 4.34 & -0.10 \\
\hline 335 & Cibicides & 134.83 & 4.29 & -0.04 \\
\hline 341 & Cibicides & 137.32 & 4.35 & 0.02 \\
\hline 341 & Cibicides & 137.32 & 4.11 & -0.08 \\
\hline 345 & Cibicides & 139.02 & 4.17 & -0.10 \\
\hline 351 & Cibicides & 141.76 & 4.12 & -0.06 \\
\hline 355 & Cibicides & 143.65 & 4.51 & -0.17 \\
\hline 361 & Cibicides & 146.63 & 4.45 & -0.14 \\
\hline 361 & Cibicides & 146.63 & 4.48 & -0.14 \\
\hline 361 & Cibicides & 146.63 & 4.35 & -0.10 \\
\hline 366 & Cibicides & 149.16 & 4.42 & -0.12 \\
\hline 371 & Cibicides & 151.71 & 4.28 & -0.16 \\
\hline 371 & Cibicides & 151.71 & 4.39 & -0.06 \\
\hline 376 & Cibicides & 154.28 & 4.40 & -0.13 \\
\hline 380 & Cibicides & 156.34 & 4.17 & -0.12 \\
\hline 386 & Cibicides & 159.46 & 4.29 & -0.11 \\
\hline 391 & Cibicides & 162.12 & 4.30 & -0.20 \\
\hline 396 & Cibicides & 164.88 & 4.35 & -0.17 \\
\hline 402 & Cibicides & 168.35 & 4.18 & -0.25 \\
\hline 402 & Cibicides & 168.35 & 4.09 & -0.13 \\
\hline 402 & Cibicides & 168.35 & 4.01 & -0.17 \\
\hline 406 & Cibicides & 170.74 & 3.97 & -0.19 \\
\hline 411 & Cibicides & 173.82 & 4.12 & -0.10 \\
\hline 416 & Cibicides & 176.94 & 4.21 & -0.19 \\
\hline 421 & Cibicides & 180.01 & 4.13 & -0.29 \\
\hline 421 & Cibicides & 180.01 & 4.02 & -0.17 \\
\hline
\end{tabular}


TABLE 1. (continued)

\begin{tabular}{|c|c|c|c|c|}
\hline Depth, cm & Genus & Age, ka & $818 \mathrm{O}$ & $\delta^{13} \mathrm{C}$ \\
\hline 426 & Cibicides & 183.03 & 4.00 & -0.13 \\
\hline 431 & Cibicides & 185.92 & 3.73 & -0.13 \\
\hline 436 & Cibicides & 188.78 & 3.70 & 0.06 \\
\hline 440 & Cibicides & 191.04 & 4.04 & -0.09 \\
\hline 440 & Cibicides & 191.04 & 3.37 & 0.09 \\
\hline 440 & Cibicides & 191.04 & 3.43 & -0.07 \\
\hline 446 & Cibicides & 194.70 & 3.40 & 0.24 \\
\hline 451 & Cibicides & 198.01 & 3.44 & 0.12 \\
\hline 455 & Cibicides & 200.90 & 3.29 & 0.10 \\
\hline 461 & Cibicides & 205.75 & 3.33 & 0.08 \\
\hline 461 & Cibicides & 205.75 & 3.27 & 0.05 \\
\hline 461 & Cibicides & 205.75 & 3.22 & 0.17 \\
\hline 465 & Cibicides & 209.09 & 3.65 & 0.15 \\
\hline 471 & Cibicides & 214.09 & 3.62 & 0.03 \\
\hline 475 & Cibicides & 217.33 & 3.80 & 0.07 \\
\hline 480 & Cibicides & 221.17 & 3.84 & -0.24 \\
\hline 481 & Cibicides & 221.88 & 3.99 & -0.04 \\
\hline 485 & Cibicides & 224.75 & 4.10 & -0.26 \\
\hline 496 & Cibicides & 231.99 & 3.76 & -0.37 \\
\hline 501 & Cibicides & 235.04 & 3.99 & -0.27 \\
\hline 501 & Cibicides & 235.04 & 3.85 & -0.12 \\
\hline 506 & Cibicides & 237.95 & 3.69 & -0.08 \\
\hline 511 & Cibicides & 240.74 & 3.88 & -0.16 \\
\hline 516 & Cibicides & 243.43 & 3.61 & -0.11 \\
\hline 520 & Cibicides & 245.55 & 4.08 & -0.21 \\
\hline 520 & Cibicides & 245.55 & 4.11 & 0.06 \\
\hline 520 & Cibicides & 245.55 & 3.65 & 0.06 \\
\hline 526 & Cibicides & 248.64 & 4.18 & -0.14 \\
\hline 531 & Cibicides & 251.22 & 4.19 & -0.18 \\
\hline 536 & Cibicides & 253.75 & 4.10 & -0.22 \\
\hline 541 & Cibicides & 256.36 & 4.22 & -0.39 \\
\hline 541 & Cibicides & 256.36 & 4.22 & -0.16 \\
\hline 541 & Cibicides & 256.36 & 4.16 & -0.24 \\
\hline 545 & Cibicides & 258.50 & 4.17 & -0.23 \\
\hline 550 & Cibicides & 261.23 & 4.02 & -0.27 \\
\hline 556 & Cibicides & 264.61 & 4.14 & -0.26 \\
\hline 561 & Cibicides & 267.43 & 4.00 & -0.07 \\
\hline 561 & Cibicides & 267.43 & 4.20 & -0.11 \\
\hline 561 & Cibicides & 267.43 & 4.13 & -0.19 \\
\hline 566 & Cibicides & 270.19 & 3.90 & -0.19 \\
\hline 571 & Cibicides & 272.86 & 4.04 & -0.30 \\
\hline 576 & Cibicides & 275.40 & 4.04 & -0.21 \\
\hline 581 & Cibicides & 277.78 & 3.58 & -0.33 \\
\hline 581 & Cibicides & 277.78 & 3.98 & -0.21 \\
\hline 581 & Cibicides & 277.78 & 3.55 & -0.23 \\
\hline 585 & Cibicides & 279.58 & 4.00 & -0.18 \\
\hline 591 & Cibicides & 282.04 & 3.67 & -0.11 \\
\hline 596 & Cibicides & 283.92 & 3.38 & 0.04 \\
\hline 600 & Cibicides & 285.31 & 3.41 & -0.15 \\
\hline 600 & Cibicides & 285.31 & 3.65 & 0.06 \\
\hline 600 & Cibicides & 285.31 & 3.43 & -0.05 \\
\hline 606 & Cibicides & 287.21 & 3.50 & 0.01 \\
\hline 611 & Cibicides & 288.67 & 3.65 & 0.06 \\
\hline 615 & Cibicides & 289.76 & 3.47 & -0.01 \\
\hline 621 & Cibicides & 291.28 & 3.85 & -0.22 \\
\hline 621 & Cibicides & 291.28 & 3.80 & -0.25 \\
\hline
\end{tabular}


TABLE 1. (continued)

\begin{tabular}{|c|c|c|c|c|}
\hline Depth, cm & Genus & Age, ka & $\delta 180$ & $\delta 13 \mathrm{C}$ \\
\hline 621 & Cibicides & 291.28 & 4.03 & -0.15 \\
\hline 626 & Cibicides & 292.48 & 3.93 & -0.06 \\
\hline 631 & Cibicides & 293.62 & 3.89 & -0.20 \\
\hline 636 & Cibicides & 294.71 & 3.93 & -0.20 \\
\hline 641 & Cibicides & 295.77 & 3.90 & -0.17 \\
\hline 641 & Cibicides & 295.77 & 3.88 & -0.30 \\
\hline 641 & Cibicides & 295.77 & 3.83 & -0.20 \\
\hline 646 & Cibicides & 296.81 & 3.82 & -0.17 \\
\hline 651 & Cibicides & 297.84 & 3.80 & -0.17 \\
\hline 656 & Cibicides & 298.87 & 3.83 & -0.05 \\
\hline 661 & Cibicides & 299.90 & 3.65 & -0.14 \\
\hline 661 & Cibicides & 299.90 & 3.68 & -0.07 \\
\hline 666 & Cibicides & 300.95 & 3.67 & -0.20 \\
\hline 671 & Cibicides & 302.02 & 3.73 & -0.16 \\
\hline 676 & Cibicides & 303.12 & 4.03 & -0.20 \\
\hline 681 & Cibicides & 304.26 & 3.60 & -0.22 \\
\hline 681 & Cibicides & 304.26 & 3.65 & -0.10 \\
\hline 686 & Cibicides & 305.46 & 3.61 & -0.12 \\
\hline 691 & Cibicides & 306.72 & 3.69 & -0.08 \\
\hline 696 & Cibicides & 308.08 & 3.58 & -0.10 \\
\hline 702 & Cibicides & 309.85 & 3.45 & -0.23 \\
\hline 702 & Cibicides & 309.85 & 3.37 & -0.26 \\
\hline 706 & Cibicides & 311.11 & 3.21 & -0.02 \\
\hline 706 & Cibicides & 311.11 & 3.49 & 0.01 \\
\hline 711 & Cibicides & 312.82 & 3.37 & -0.11 \\
\hline 716 & Cibicides & 314.67 & 3.28 & -0.01 \\
\hline 721 & Cibicides & 316.68 & 3.34 & -0.18 \\
\hline 721 & Cibicides & 316.68 & 3.19 & 0.04 \\
\hline 726 & Cibicides & 318.82 & 3.29 & 0.03 \\
\hline 731 & Cibicides & 321.09 & 3.34 & 0.15 \\
\hline 736 & Cibicides & 323.40 & 3.14 & 0.05 \\
\hline 741 & Cibicides & 325.70 & 3.03 & 0.01 \\
\hline 741 & Cibicides & 325.70 & 2.97 & 0.14 \\
\hline 746 & Cibicides & 327.88 & 3.16 & -0.03 \\
\hline 751 & Cibicides & 329.88 & 2.96 & 0.07 \\
\hline 756 & Cibicides & 331.64 & 3.20 & 0.02 \\
\hline 761 & Cibicides & 333.13 & 2.63 & -0.10 \\
\hline 761 & Cibicides & 333.13 & 2.89 & -0.09 \\
\hline 766 & Cibicides & 334.37 & 3.06 & 0.09 \\
\hline 766 & Cibicides & 334.37 & 3.05 & -0.11 \\
\hline 771 & Cibicides & 335.40 & 3.62 & -0.16 \\
\hline 776 & Cibicides & 336.26 & 3.43 & -0.17 \\
\hline 781 & Cibicides & 337.02 & 3.92 & -0.26 \\
\hline 786 & Cibicides & 337.73 & 3.65 & -0.31 \\
\hline 791 & Cibicides & 338.46 & 3.95 & -0.43 \\
\hline 796 & Cibicides & 339.27 & 4.19 & -0.24 \\
\hline 801 & Cibicides & 340.19 & 3.94 & -0.22 \\
\hline 801 & Cibicides & 340.19 & 3.70 & -0.22 \\
\hline 806 & Cibicides & 341.24 & 3.97 & -0.37 \\
\hline 810 & Cibicides & 342.18 & 4.07 & -0.26 \\
\hline 816 & Cibicides & 343.73 & 4.46 & -0.24 \\
\hline 821 & Cibicides & 345.06 & 4.33 & -0.44 \\
\hline 821 & Cibicides & 345.06 & 4.43 & -0.23 \\
\hline 826 & Cibicides & 346.36 & 4.43 & -0.37 \\
\hline 831 & Cibicides & 347.59 & 4.52 & -0.39 \\
\hline 836 & Cibicides & 348.73 & 4.28 & -0.28 \\
\hline
\end{tabular}


TABLE 1. (continued)

\begin{tabular}{cllll}
\hline Depth, $\mathrm{cm}$ & Genus & Age, $\mathrm{ka}$ & $\delta 18 \mathrm{O}$ & $\delta 13 \mathrm{C}$ \\
\hline 840 & & & & \\
846 & Cibicides & 349.60 & 4.29 & -0.39 \\
851 & Cibicides & 350.81 & 4.19 & -0.47 \\
856 & Cibicides & 351.79 & 3.84 & -0.42 \\
861 & Cibicides & 352.77 & 4.08 & -0.44 \\
861 & Cibicides & 353.75 & 4.04 & -0.30 \\
866 & Cibicides & 353.75 & 3.96 & -0.20 \\
871 & Cibicides & 354.77 & 4.05 & -0.33 \\
876 & Cibicides & 355.80 & 3.91 & -0.24 \\
881 & Cibicides & 356.87 & 4.21 & -0.19 \\
881 & Cibicides & 357.99 & 4.16 & -0.24 \\
886 & Cibicides & 357.99 & 4.10 & -0.11 \\
891 & Cibicides & 359.16 & 4.10 & -0.28 \\
896 & Cibicides & 360.40 & 4.06 & -0.36 \\
905 & Cibicides & 361.70 & 4.08 & -0.34 \\
909 & Cibicides & 364.21 & 3.84 & -0.13 \\
909 & Cibicides & 365.35 & 3.87 & -0.70 \\
911 & Cibicides & 365.35 & 3.89 & -0.60 \\
916 & Cibicides & 365.93 & 3.85 & 0.00 \\
921 & Cibicides & 367.40 & 3.80 & -0.08 \\
926 & Cibicides & 368.86 & 3.76 & -0.09 \\
931 & Cibicides & 370.33 & 4.01 & -0.01 \\
937 & Cibicides & 371.80 & 3.80 & 0.04 \\
942 & Cibicides & 373.00 & 3.98 & -0.08 \\
942 & Cibicides & 374.00 & 3.86 & -0.10 \\
946 & Cibicides & 374.00 & 3.73 & -0.01 \\
951 & Cibicides & 374.80 & 3.74 & -0.15 \\
& Cibicides & 377.80 & 3.67 & 0.01
\end{tabular}

Core $\mathrm{RC} 13-1100.1^{\circ} \mathrm{N}, 95.65^{\circ} \mathrm{W}, 3231 \mathrm{~m}$ Depth

$\begin{array}{rlrrr}1 & \text { Cibicides } & 0.36 & 3.39 & \\ 11 & \text { Cibicides } & 3.88 & 3.23 & 0.18 \\ 15 & \text { Cibicides } & 5.01 & 3.49 & 0.10 \\ 21 & \text { Cibicides } & 6.96 & 3.50 & 0.12 \\ 24 & \text { Cibicides } & 7.97 & 3.91 & -0.05 \\ 31 & \text { Cibicides } & 10.33 & 4.24 & -0.14 \\ 34 & \text { Cibicides } & 11.33 & 4.01 & -0.11 \\ 35 & \text { Cibicides } & 11.66 & 4.60 & -0.40 \\ 41 & \text { Cibicides } & 13.64 & 4.22 & -0.13 \\ 44 & \text { Cibicides } & 14.66 & 4.13 & -0.25 \\ 51 & \text { Cibicides } & 16.96 & 4.70 & -0.39 \\ 54 & \text { Uvigerina } & 17.94 & 4.83 & -0.17 \\ 60 & \text { Cibicides } & 19.84 & 4.73 & -0.23 \\ 64 & \text { Cibicides } & 21.08 & 4.85 & -0.28 \\ 70 & \text { Cibicides } & 22.85 & 4.60 & -0.20 \\ 74 & \text { Cibicides } & 24.00 & 4.54 & -0.27 \\ 81 & \text { Cibicides } & 25.98 & 4.43 & -0.14 \\ 84 & \text { Cibicides } & 26.86 & 4.53 & -0.21 \\ 91 & \text { Cibicides } & 28.97 & 4.34 & -0.03 \\ 94 & \text { Cibicides } & 29.96 & 4.50 & -0.13 \\ 101 & \text { Uvigerina } & 32.46 & 4.31 & -0.04 \\ 111 & \text { Cibicides } & 36.44 & 4.45 & 0.03 \\ 114 & \text { Cibicides } & 37.70 & 4.32 & 0.00 \\ 121 & \text { Cibicides } & 40.55 & 4.31 & -0.09 \\ 124 & \text { Cibicides } & 41.78 & 4.56 & 0.02 \\ 131 & \text { Cibicides } & 44.58 & 4.22 & 0.04\end{array}$


TABLE 1. (continued)

\begin{tabular}{|c|c|c|c|c|}
\hline Depth, $\mathrm{cm}$ & Genus & Age, $\mathrm{ka}$ & $\delta 180$ & $\delta 13 \mathrm{C}$ \\
\hline 134 & Cibicides & 45.80 & 4.43 & -0.03 \\
\hline 141 & Cibicides & 48.54 & 4.21 & -0.12 \\
\hline 144 & Cibicides & 49.72 & 4.45 & -0.15 \\
\hline 154 & Cibicides & 53.41 & 4.44 & -0.09 \\
\hline 161 & Cibicides & 55.82 & 4.33 & -0.28 \\
\hline 171 & Cibicides & 59.24 & 4.47 & -0.61 \\
\hline 175 & Cibicides & 60.70 & 4.34 & -0.13 \\
\hline 181 & Cibicides & 63.17 & 4.49 & -0.26 \\
\hline 184 & Cibicides & 64.62 & 4.68 & -0.35 \\
\hline 191 & Uvigerina & 68.56 & 4.63 & -0.22 \\
\hline 194 & Cibicides & 70.55 & 4.62 & -0.30 \\
\hline 201 & Cibicides & 75.72 & 3.90 & 0.01 \\
\hline 206 & Uvigerina & 79.52 & 3.88 & 0.03 \\
\hline 211 & Cibicides & 82.99 & 3.98 & 0.07 \\
\hline 214 & Uvigerina & 84.92 & 3.98 & 0.01 \\
\hline 221 & Cibicides & 89.01 & 3.80 & -0.01 \\
\hline 224 & Cibicides & 90.66 & 3.95 & 0.13 \\
\hline 241 & Cibicides & 99.34 & 3.72 & -0.29 \\
\hline 251 & Cibicides & 103.74 & 3.89 & -0.14 \\
\hline 254 & Cibicides & 105.11 & 3.83 & -0.17 \\
\hline 261 & Cibicides & 108.61 & 3.97 & -0.13 \\
\hline 263 & Cibicides & 109.75 & 3.85 & -0.07 \\
\hline 271 & Cibicides & 114.39 & 3.66 & 0.05 \\
\hline 275 & Cibicides & 116.68 & 3.15 & -0.26 \\
\hline 281 & Cibicides & 119.85 & 3.07 & -0.01 \\
\hline 284 & Cibicides & 121.36 & 3.21 & -0.08 \\
\hline 291 & Cibicides & 124.52 & 3.32 & -0.19 \\
\hline 301 & Cibicides & 128.52 & 3.71 & -0.05 \\
\hline 305 & Cibicides & 130.06 & 4.38 & -0.23 \\
\hline 311 & Cibicides & 132.37 & 4.50 & -0.35 \\
\hline 314 & Cibicides & 133.63 & 4.80 & -0.41 \\
\hline 321 & Cibicides & 136.84 & 4.79 & -0.33 \\
\hline 324 & Cibicides & 138.38 & 4.72 & -0.47 \\
\hline 331 & Cibicides & 142.16 & 4.67 & -0.55 \\
\hline 334 & Cibicides & 143.86 & 4.80 & -0.43 \\
\hline 341 & Cibicides & 147.63 & 4.62 & -0.44 \\
\hline 344 & Cibicides & 149.18 & 4.91 & -0.37 \\
\hline 351 & Cibicides & 152.46 & 4.59 & -0.39 \\
\hline 354 & Cibicides & 153.75 & 4.71 & -0.46 \\
\hline 361 & Cibicides & 156.43 & 4.62 & -0.56 \\
\hline 364 & Cibicides & 157.53 & 4.78 & -0.54 \\
\hline 371 & Cibicides & 159.93 & 4.60 & -0.50 \\
\hline 374 & Cibicides & 160.98 & 4.70 & -0.38 \\
\hline 384 & Cibicides & 164.44 & 4.51 & -0.60 \\
\hline 391 & Cibicides & 166.99 & 4.51 & -0.46 \\
\hline 394 & Cibicides & 168.20 & 4.41 & -0.51 \\
\hline 404 & Cibicides & 172.78 & 4.41 & -0.50 \\
\hline 414 & Cibicides & 178.24 & 4.44 & -0.46 \\
\hline 421 & Cibicides & 182.17 & 4.46 & -0.42 \\
\hline 424 & Cibicides & 183.86 & 4.36 & -0.51 \\
\hline 431 & Cibicides & 187.66 & 4.31 & -0.45 \\
\hline 434 & Cibicides & 189.34 & 3.76 & 0.02 \\
\hline 441 & Cibicides & 193.28 & 3.50 & -0.11 \\
\hline 443 & Cibicides & 194.48 & 3.63 & -0.05 \\
\hline 451 & Cibicides & 199.45 & 3.58 & -0.10 \\
\hline 461 & Cibicides & 206.03 & 3.54 & 0.02 \\
\hline
\end{tabular}


TABLE 1. (continued)

\begin{tabular}{|c|c|c|c|c|}
\hline Depth, cm & Genus & Age, $\mathrm{ka}$ & 8180 & $\delta^{13} \mathrm{C}$ \\
\hline 464 & Cibicides & 207.99 & 3.82 & -0.12 \\
\hline 471 & Uvigerina & 212.24 & 3.85 & -0.28 \\
\hline 475 & Cibicides & 214.55 & 3.68 & -0.15 \\
\hline 481 & Cibicides & 217.64 & 3.70 & -0.24 \\
\hline 485 & Cibicides & 219.62 & 3.92 & -0.30 \\
\hline 491 & Cibicides & 222.29 & 3.67 & -0.22 \\
\hline 494 & Cibicides & 223.60 & 4.19 & -0.52 \\
\hline 501 & Cibicides & 226.40 & 4.00 & -0.46 \\
\hline 504 & Uvigerina & 227.57 & 4.26 & -0.64 \\
\hline 511 & Uvigerina & 230.12 & 4.07 & -0.47 \\
\hline 514 & Cibicides & 231.18 & 3.97 & -0.22 \\
\hline 521 & Cibicides & 233.54 & 4.01 & -0.29 \\
\hline 524 & Cibicides & 234.53 & 4.00 & -0.18 \\
\hline 531 & Cibicides & 236.74 & 3.62 & -0.08 \\
\hline 534 & Cibicides & 237.67 & 3.65 & 0.03 \\
\hline 541 & Cibicides & 239.72 & 3.43 & -0.13 \\
\hline 544 & Cibicides & 240.60 & 3.83 & -0.22 \\
\hline 551 & Cibicides & 242.64 & 3.71 & -0.41 \\
\hline 555 & Cibicides & 243.89 & 4.01 & -0.39 \\
\hline 561 & Cibicides & 245.97 & 3.54 & -0.61 \\
\hline 565 & Cibicides & 247.61 & 4.63 & -0.42 \\
\hline 571 & Cibicides & 250.58 & 4.15 & -0.56 \\
\hline 575 & Cibicides & 252.92 & 4.62 & -0.43 \\
\hline 581 & Cibicides & 257.06 & 4.21 & -0.64 \\
\hline 585 & Cibicides & 260.13 & 4.49 & -0.81 \\
\hline 591 & Cibicides & 264.89 & 4.35 & -0.76 \\
\hline 594 & Uvigerina & 267.30 & 4.62 & -0.64 \\
\hline 601 & Cibicides & 272.66 & 4.01 & -0.63 \\
\hline 605 & Cibicides & 275.59 & 4.39 & -0.71 \\
\hline 611 & Cibicides & 279.68 & 4.02 & -0.10 \\
\hline 621 & Cibicides & 286.08 & 3.82 & -0.30 \\
\hline 631 & Cibicides & 291.89 & 3.97 & -0.36 \\
\hline 633 & Uvigerina & 293.00 & 4.09 & -0.17 \\
\hline 641 & Cibicides & 297.21 & 4.10 & -0.31 \\
\hline 645 & Cibicides & 299.30 & 4.14 & -0.36 \\
\hline 651 & Cibicides & 302.51 & 3.97 & -0.27 \\
\hline 653 & Cibicides & 303.64 & 4.12 & -0.23 \\
\hline 661 & Uvigerina & 308.31 & 3.69 & -0.01 \\
\hline 665 & Cibicides & 310.86 & 3.70 & -0.17 \\
\hline 675 & Cibicides & 317.46 & 3.72 & -0.21 \\
\hline 681 & Cibicides & 321.25 & 3.35 & -0.03 \\
\hline 685 & Cibicides & 323.67 & 3.49 & -0.11 \\
\hline 691 & Uvigerina & 326.88 & 3.11 & 0.13 \\
\hline 694 & Cibicides & 328.34 & 3.20 & -0.14 \\
\hline 701 & Uvigerina & 331.20 & 3.27 & 0.14 \\
\hline 705 & Cibicides & 332.59 & 3.18 & -0.34 \\
\hline 711 & Cibicides & 334.33 & 3.35 & -0.27 \\
\hline 715 & Cibicides & 335.35 & 3.65 & -0.36 \\
\hline 721 & Cibicides & 336.80 & 4.12 & -0.45 \\
\hline 725 & Cibicides & 337.78 & 4.24 & -0.40 \\
\hline 731 & Cibicides & 339.35 & 4.37 & -0.54 \\
\hline 734 & Cibicides & 340.23 & 4.63 & -0.68 \\
\hline 741 & Cibicides & 342.48 & 4.60 & -0.52 \\
\hline 745 & Cibicides & 343.97 & 4.44 & -0.54 \\
\hline 751 & Cibicides & 346.51 & 4.43 & -0.48 \\
\hline 761 & Cibicides & 351.32 & 4.40 & -0.57 \\
\hline
\end{tabular}


TABLE 1. (continued)

\begin{tabular}{|c|c|c|c|c|}
\hline Depth, cm & Genus & Age, ka & $818 \mathrm{O}$ & $\delta 13 \mathrm{C}$ \\
\hline 763 & Cibicides & 352.34 & 4.42 & -0.59 \\
\hline 771 & Cibicides & 356.39 & 4.39 & -0.46 \\
\hline 775 & Cibicides & 358.45 & 4.23 & -0.54 \\
\hline 781 & Cibicides & 361.51 & 4.06 & -0.58 \\
\hline 785 & Uvigerina & 363.64 & 4.27 & -0.22 \\
\hline 791 & Cibicides & 366.94 & 4.02 & -0.22 \\
\hline 794 & Cibicides & 368.69 & 4.11 & -0.37 \\
\hline 801 & Cibicides & 372.81 & 4.01 & -0.31 \\
\hline 805 & Uvigerina & 375.29 & 4.18 & 0.16 \\
\hline 811 & Uvigerina & 379.02 & 3.65 & -0.17 \\
\hline 814 & Uvigerina & 380.94 & 4.04 & 0.15 \\
\hline 821 & Cibicides & 385.44 & 3.54 & -0.14 \\
\hline 825 & Uvigerina & 388.05 & 3.67 & 0.04 \\
\hline 831 & Cibicides & 391.90 & 3.60 & -0.04 \\
\hline 835 & Cibicides & 394.48 & 3.31 & 0.27 \\
\hline 841 & Cibicides & 398.18 & 2.92 & 0.43 \\
\hline 845 & Cibicides & 400.61 & 3.23 & 0.29 \\
\hline 851 & Cibicides & 404.10 & 3.03 & 0.22 \\
\hline 854 & Uvigerina & 405.81 & 3.19 & 0.45 \\
\hline 861 & Cibicides & 409.52 & 3.09 & 0.22 \\
\hline 864 & Cibicides & 411.06 & 3.42 & 0.10 \\
\hline 871 & Uvigerina & 414.53 & 3.19 & 0.31 \\
\hline 873 & Uvigerina & 415.51 & 3.52 & -0.20 \\
\hline 881 & Uvigerina & 419.45 & 3.91 & -0.13 \\
\hline 891 & Uvigerina & 424.41 & 4.08 & -0.21 \\
\hline 894 & Uvigerina & 425.89 & 4.68 & -0.42 \\
\hline 901 & Cibicides & 429.25 & 4.22 & -0.32 \\
\hline 905 & Uvigerina & 431.19 & 4.85 & -0.40 \\
\hline 911 & Uvigerina & 434.06 & 4.87 & -0.21 \\
\hline 914 & Uvigerina & 435.54 & 4.92 & -0.26 \\
\hline 921 & Cibicides & 438.99 & 4.42 & -0.34 \\
\hline 924 & Uvigerina & 440.52 & 4.77 & -0.13 \\
\hline 931 & Uvigerina & 444.01 & 4.65 & -0.24 \\
\hline 934 & Uvigerina & 445.53 & 4.89 & -0.06 \\
\hline 941 & Cibicides & 449.02 & 4.42 & -0.41 \\
\hline 945 & Uvigerina & 451.04 & 4.54 & -0.30 \\
\hline 951 & Cibicides & 454.10 & 4.10 & -0.25 \\
\hline 954 & Cibicides & 455.66 & 4.67 & -0.48 \\
\hline 961 & Cibicides & 459.29 & 4.32 & -0.54 \\
\hline 965 & Uvigerina & 461.41 & 4.38 & -0.27 \\
\hline 971 & Uvigerina & 464.43 & 4.24 & -0.07 \\
\hline 975 & Cibicides & 466.39 & 4.55 & -0.41 \\
\hline 981 & Cibicides & 469.00 & 4.47 & -0.22 \\
\hline 985 & Uvigerina & 470.59 & 4.34 & -0.27 \\
\hline 991 & Cibicides & 472.63 & 4.36 & -0.30 \\
\hline 995 & Cibicides & 473.86 & 4.31 & -0.26 \\
\hline 1001 & Cibicides & 475.45 & 4.30 & -0.09 \\
\hline 1005 & Uvigerina & 476.49 & 4.05 & -0.09 \\
\hline 1011 & Uvigerina & 478.14 & 4.34 & 0.11 \\
\hline 1015 & Uvigerina & 479.38 & 4.07 & 0.10 \\
\hline 1025 & Cibicides & 483.22 & 3.30 & 0.14 \\
\hline 1031 & Cibicides & 486.17 & 3.61 & 0.36 \\
\hline 1035 & Uvigerina & 488.44 & 3.21 & 0.53 \\
\hline 1041 & Cibicides & 492.40 & 3.73 & 0.32 \\
\hline 1051 & Cibicides & 500.07 & 3.72 & 0.29 \\
\hline 1054 & Uvigerina & 502.51 & 3.51 & 0.30 \\
\hline
\end{tabular}


TABLE 1. (continued)

\begin{tabular}{|c|c|c|c|c|}
\hline Depth, cm & Genus & Age, ka & $\delta 180$ & $\delta^{13} \mathrm{C}$ \\
\hline 1061 & Cibicides & 508.08 & 4.09 & 0.06 \\
\hline 1065 & Uvigerina & 511.14 & 4.27 & 0.42 \\
\hline 1071 & Cibicides & 515.65 & 4.14 & 0.32 \\
\hline 1075 & Uvigerina & 518.68 & 4.08 & 0.10 \\
\hline 1081 & Cibicides & 523.31 & 3.90 & 0.17 \\
\hline 1085 & Uvigerina & 526.60 & 3.90 & 0.40 \\
\hline 1091 & Cibicides & 531.76 & 3.87 & 0.10 \\
\hline 1095 & Cibicides & 535.50 & 4.08 & -0.01 \\
\hline 1101 & Uvigerina & 541.34 & 3.83 & 0.05 \\
\hline 1111 & Uvigerina & 551.11 & 4.27 & -0.13 \\
\hline 1114 & Uvigerina & 553.82 & 4.45 & -0.14 \\
\hline 1121 & Uvigerina & 559.36 & 4.39 & -0.27 \\
\hline 1125 & Uvigerina & 562.09 & 4.50 & -0.03 \\
\hline 1134 & Uvigerina & 567.02 & 4.32 & -0.23 \\
\hline 1141 & Uvigerina & 570.28 & 3.88 & -0.11 \\
\hline 1145 & Uvigerina & 572.07 & 4.16 & -0.09 \\
\hline 1155 & Cibicides & 576.32 & 3.86 & -0.16 \\
\hline 1155 & Uvigerina & 576.32 & 4.14 & 0.09 \\
\hline 1161 & Uvigerina & 578.84 & 3.79 & -0.03 \\
\hline 1165 & Cibicides & 580.53 & 3.81 & -0.31 \\
\hline 1171 & Uvigerina & 583.08 & 3.89 & -0.21 \\
\hline 1181 & Cibicides & 587.29 & 3.99 & -0.04 \\
\hline 1185 & Cibicides & 588.97 & 3.98 & -0.14 \\
\hline 1191 & Uvigerina & 591.72 & 3.98 & 0.02 \\
\hline 1194 & Cibicides & 593.23 & 3.92 & -0.21 \\
\hline 1194 & Uvigerina & 593.23 & 3.93 & 0.07 \\
\hline 1201 & Uvigerina & 597.04 & 3.91 & 0.06 \\
\hline 1205 & Cibicides & 599.48 & 3.83 & -0.11 \\
\hline 1205 & Uvigerina & 599.48 & 3.68 & 0.10 \\
\hline 1210 & Uvigerina & 602.71 & 3.82 & 0.15 \\
\hline 1214 & Cibicides & 605.48 & 3.92 & -0.09 \\
\hline 1214 & Uvigerina & 605.48 & 3.85 & 0.38 \\
\hline 1220 & Uvigerina & 609.74 & 3.86 & 0.23 \\
\hline 1234 & Cibicides & 618.34 & 4.16 & -0.16 \\
\hline 1244 & Cibicides & 622.57 & 4.88 & -0.34 \\
\hline 1261 & Cibicides & 629.68 & 4.90 & -0.38 \\
\hline 1265 & Cibicides & 631.43 & 4.87 & -0.48 \\
\hline 1265 & Uvigerina & 631.43 & 4.76 & -0.39 \\
\hline 1271 & Cibicides & 633.99 & 4.87 & -0.40 \\
\hline 1275 & Cibicides & 635.80 & 4.80 & -0.53 \\
\hline 1275 & Uvigerina & 635.80 & 4.84 & -0.41 \\
\hline 1281 & Cibicides & 638.72 & 4.57 & -0.43 \\
\hline 1291 & Cibicides & 644.01 & 4.52 & -0.47 \\
\hline 1294 & Cibicides & 645.56 & 4.62 & -0.53 \\
\hline 1294 & Uvigerina & 645.56 & 4.73 & -0.41 \\
\hline 1301 & Cibicides & 649.04 & 4.46 & -0.52 \\
\hline 1304 & Uvigerina & 650.53 & 4.56 & -0.45 \\
\hline 1311 & Uvigerina & 653.98 & 4.35 & -0.69 \\
\hline 1314 & Uvigerina & 655.48 & 4.82 & -0.43 \\
\hline 1321 & Uvigerina & 658.99 & 4.34 & -0.48 \\
\hline 1325 & Cibicides & 661.05 & 4.40 & -0.68 \\
\hline 1325 & Uvigerina & 661.05 & 4.61 & -0.41 \\
\hline 1331 & Cibicides & 664.00 & 4.29 & -0.60 \\
\hline 1334 & Cibicides & 665.50 & 4.44 & -0.54 \\
\hline 1334 & Uvigerina & 665.50 & 4.50 & -0.56 \\
\hline 1341 & Uvigerina & 668.93 & 4.36 & -0.43 \\
\hline
\end{tabular}


TABLE 1. (continued)

\begin{tabular}{|c|c|c|c|c|}
\hline Depth, cm & Genus & Age, ka & $\delta 180$ & $\delta 13 \mathrm{C}$ \\
\hline 1344 & Cibicides & 670.44 & 4.40 & -0.62 \\
\hline 1345 & Cibicides & 670.94 & 4.40 & -0.48 \\
\hline 1351 & Cibicides & 673.92 & 4.05 & -0.39 \\
\hline 1361 & Uvigerina & 678.93 & 4.04 & -0.32 \\
\hline 1364 & Uvigerina & 680.38 & 3.93 & -0.31 \\
\hline 1371 & Cibicides & 683.55 & 3.57 & -0.32 \\
\hline 1374 & Cibicides & 684.81 & 3.69 & -0.08 \\
\hline 1381 & Cibicides & 687.43 & 3.52 & 0.01 \\
\hline 1384 & Cibicides & 688.44 & 3.59 & -0.13 \\
\hline 1391 & Cibicides & 690.49 & 3.58 & -0.09 \\
\hline 1394 & Uvigerina & 691.28 & 3.77 & -0.13 \\
\hline 1401 & Cibicides & 692.90 & 4.47 & -0.08 \\
\hline 1404 & Cibicides & 693.57 & 3.98 & -0.16 \\
\hline 1411 & Cibicides & 695.10 & 4.74 & -0.53 \\
\hline 1421 & Cibicides & 697.44 & 5.17 & -0.40 \\
\hline 1424 & Uvigerina & 698.18 & 4.49 & -0.49 \\
\hline 1431 & Cibicides & 699.95 & 5.07 & -0.48 \\
\hline 1434 & Uvigerina & 700.74 & 4.60 & -0.64 \\
\hline 1441 & Cibicides & 702.61 & 5.00 & -0.41 \\
\hline 1444 & Uvigerina & 703.45 & 4.27 & -0.37 \\
\hline 1451 & Cibicides & 705.41 & 4.34 & -0.18 \\
\hline 1454 & Uvigerina & 706.28 & 4.01 & -0.19 \\
\hline 1461 & Uvigerina & 708.28 & 4.14 & -0.30 \\
\hline 1464 & Uvigerina & 709.18 & 4.07 & -0.22 \\
\hline 1471 & Cibicides & 711.27 & 4.11 & -0.28 \\
\hline 1474 & Cibicides & 712.21 & 4.21 & -0.57 \\
\hline 1481 & Uvigerina & 714.41 & 4.06 & -0.13 \\
\hline 1484 & Uvigerina & 715.40 & 4.27 & -0.56 \\
\hline 1491 & Uvigerina & 717.68 & 4.23 & -0.48 \\
\hline 1494 & Uvigerina & 718.68 & 4.36 & -0.54 \\
\hline 1501 & Cibicides & 721.00 & 4.51 & -0.50 \\
\hline 1505 & Uvigerina & 722.32 & 4.31 & -0.42 \\
\hline 1511 & Cibicides & 724.30 & 4.55 & -0.54 \\
\hline 1514 & Cibicides & 725.29 & 4.32 & -0.62 \\
\hline 1514 & Uvigerina & 725.29 & 4.23 & -0.54 \\
\hline 1521 & Uvigerina & 727.59 & 4.25 & -0.50 \\
\hline 1524 & Cibicides & 728.58 & 4.09 & -0.47 \\
\hline 1531 & Cibicides & 730.89 & 4.00 & -0.41 \\
\hline 1541 & Uvigerina & 734.19 & 4.07 & -0.39 \\
\hline 1544 & Uvigerina & 735.18 & 4.01 & -0.35 \\
\hline
\end{tabular}

Isotope data are tabulated as per mil versus $\mathrm{PDB}$. Values for $\delta 18 \mathrm{O}$ are corrected to $U$ vigerina (Cibicides +0.64$)$. Values for $\delta^{13} \mathrm{C}$ are corrected to Cibicides (Uvigerina +0.90$)$. Ages are based on correlation to the SPECMAP long time scale [Imbrie et al., 1984]. T indicates samples from trigger core. All other samples are from the piston cores.

would be appropriate for the section from 600 to 900 $\mathrm{cm}$ in this core. We have not used a variable correction factor in our analysis but instead limit of data analysis to the section of core RC13-110 that is dominated by $C$. wuellerstorfi.

The $\delta 13$ C offset between $C$. whellerstorf $i$ in RC13-110 and Uvigerina in V19-30 is roughly constant (Figure $4 \mathrm{~b}$ ). There are significant anomalies near $\sim 65 \mathrm{ka}$ (stage 4), between 90 and $110 \mathrm{ka}$ (stage
5) and near $\sim 300 \mathrm{ka}$ (stage 9). These offsets may reflect true differences between the sites, local (pore water?) effects on $\delta{ }^{13} \mathrm{C}$ in Uvigerina at the site of V19-30 or errors in correlation between the sites. Modifying the time scales to force correlation between ${ }^{13} \mathrm{C}$ records would create significant mismatches in $\delta 18 \mathrm{O}$, so we reject the third possibility. At present, we can not choose from among the other options. The strong general 


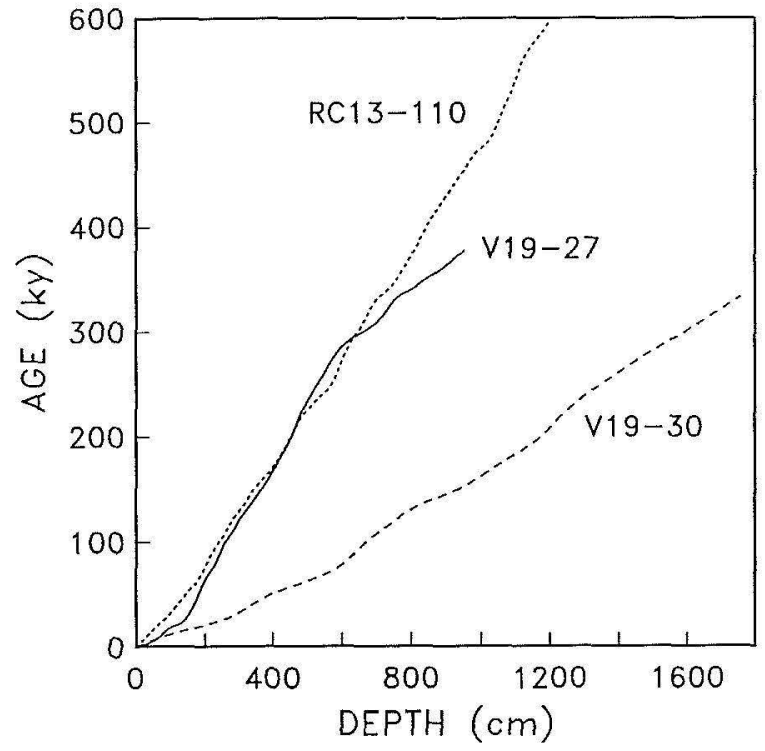

Fig. 3. Age models for cores V19-27 (solid line), V19-30 (dashed line) and RC13-110 (dotted line), by correlation to the SPECMAP $\delta 18 \mathrm{O}$ time scale [Imbrie et al., 1984].

similarity between the $\delta 13 \mathrm{C}$ records in RC13-110 and V19-30, however, suggests that we have characterized the pattern of $\delta^{13} \mathrm{C}$ variability in the deep Pacific over the last $\sim 400$ kyr. Further support comes from the stable isotope record of $C$. wuellerstorfi in Pacific core KNR73-4-3PC, from $0.28^{\circ} \mathrm{S}, 106.14^{\circ} \mathrm{W}, 3606 \mathrm{~m}$ water depth [Boyle and Keigwin, 1985/1986], which is very similar to both RC13-110 and V19-30.

To analyze intermediate-to-deep water differences in the $\delta^{13} \mathrm{C}$ records, we calculate the differences between V19-27 and RC13-110. We use core $\mathrm{RC} 13-110$ as a measure of deep-water $\delta 13 \mathrm{C}$, rather than V19-30, so that data from the preferred species, C. wuellerstorfi, are used as much as possible. Also, because both cores were analyzed in the same laboratory, we can exclude the possibility of interlaboratory calibrations producing the isotopic offsets between cores.

\section{Intermediate and Deep Water: Similarities and Differences}

The isotope data from RC13-110 and V19-27 are plotted together versus age in Figure 5. Replicate analyses shown in Figure 2 are averaged here. The well-known oxygen isotope stages 1-10 [Shackleton and Opdyke, 1973; Imbrie et al., 1984] are clearly present and easily correlated (Figure 5a).

The intermediate- and deep-water sites contain roughly similar carbon isotope signals (Figure $5 b$ ), with glacial intervals lower in $\delta 13 \mathrm{C}$ than adjacent interglacial intervals. This pattern contrasts with the
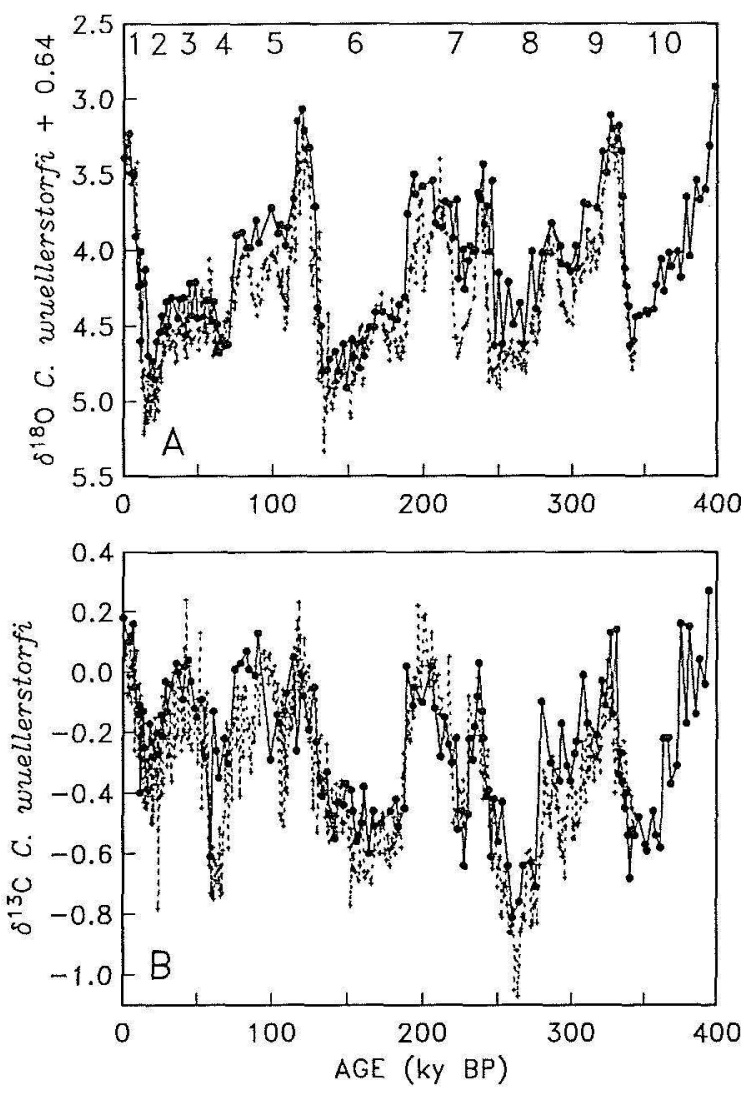

Fig. 4. Comparison of (a) $\delta^{18} \mathrm{O}$ and (b) $\delta^{13} \mathrm{C}$ records from Uvigerina sp. in V19-30 (dashed line with pluses) with those from Cibicides wuellerstorfi and Uvigerina sp. in RC13-110 (solid line and dots) plotted versus age. To facilitate comparison, $\delta 18 \mathrm{O}$ in Cibicides is adjusted by $+0.64 \%$, and $813 \mathrm{C}$ in Uvigerina is adjusted by $+0.90 \%$. Replicate analyses are averaged within depth levels.

North Atlantic and Caribbean, where in some places the intermediate-water sites are most enriched in $813 \mathrm{C}$ in the glacial intervals. Clearly, intermediate waters in the Pacific operate differently than in the Atlantic over this time frame. In spite of the first-order similarity between the sites, there are also some important differences. In RC13-110, the lowest ${ }^{813} \mathrm{C}$ values occur about $260 \mathrm{ka}$ (stage 8 ), while in V19-27 the lowest values occur about $350 \mathrm{ka}$ (stage $10)$.

To illustrate the differences in more detail, we plot the oxygen isotopic offsets $(\Delta \delta 18 \mathrm{O}$, Figure $6 \mathrm{a})$ and the carbon isotopic offsets $(\Delta \delta 13 \mathrm{C}$, Figure $6 \mathrm{~b})$ between sites (V19-27 minus RC13-110). This calculation is done at 3-kyr intervals, after interpolation and smoothing of the raw data with a Gaussian filter of 6kyr half width. For comparison, the mean $\delta 180$ record from the two cores, interpolated and smoothed in the same way, is shown in Figure $6 \mathrm{c}$ and compared 

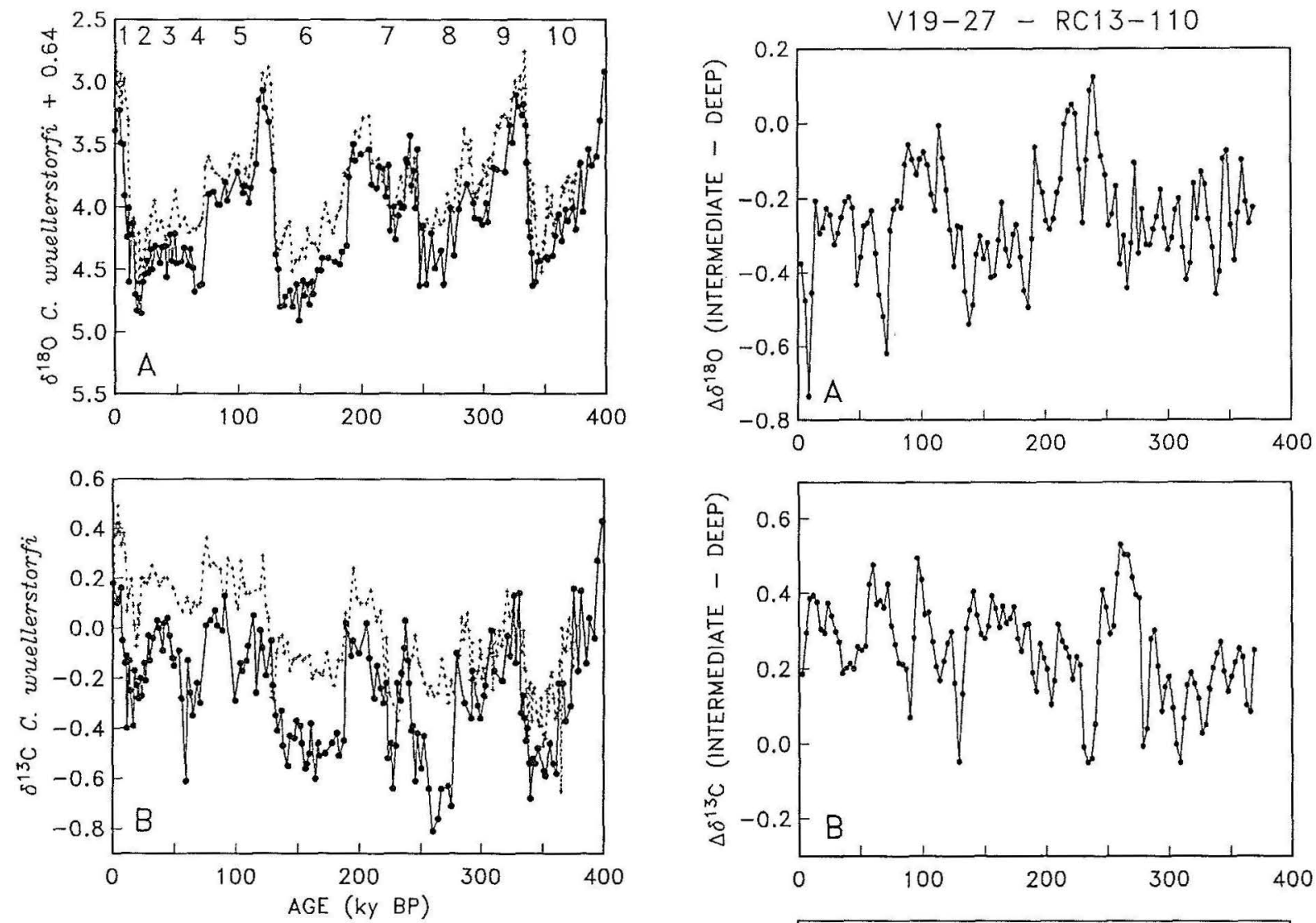

Fig. 5. (a) Oxygen isotopes and (b) carbon isotopes in V19-27 (dashed line with pluses) and RC13-110 (solid line and dots) plotted versus age. Analyses of $\delta 18 \mathrm{O}$ in Cibicides are adjusted by $+0.64 \%$, and $\delta^{13} \mathrm{C}$ in Uvigerina is adjusted by $+0.90 \%$. Replicate analyses are averaged within depth levels.

to the SPECMAP "long" $\delta 180$ time scale of Imbrie et al. [1984]. The core top $\delta 180$ offset $(\Delta \delta 18 O)$ between V19-27 and RC13-110, about $-0.4 \%$ (Figure $6 \mathrm{a}$ ), is consistent with the modern temperature difference of $\sim 2^{\circ} \mathrm{C}$ between 1400 and $3200 \mathrm{~m}$ water depth in the eastern Pacific [Levitus, 1982]. Variations in the oxygen isotope offsets between the cores are not significantly correlated with glacial-interglacial patterns, and they are not significantly coherent with orbital rhythms. For example, the stage 5 (interglacial) offset in $\delta 180$ between sites of about $0.2 \% o$ is half that of the Holocene. There appears to be a weak long-term trend to the $\delta 180$ gradient between sites, with more $\delta 180$ contrast in the younger sections. This implies greater thermal or watermass $\delta 180$ contrast between intermediate- and deep-water sites in the later Quaternary. Confirmation of this pattern should be sought in other cores.

On average, $\delta 13 \mathrm{C}$ values in V19-27 are $0.2-0.3 \%$ higher than in $\mathrm{RC} 13-110$. The average $\delta{ }^{13} \mathrm{C}$ value from the top $20 \mathrm{~cm}$ in V19-27, 0.19 $\pm 0.18 \%$ o $(n=5)$

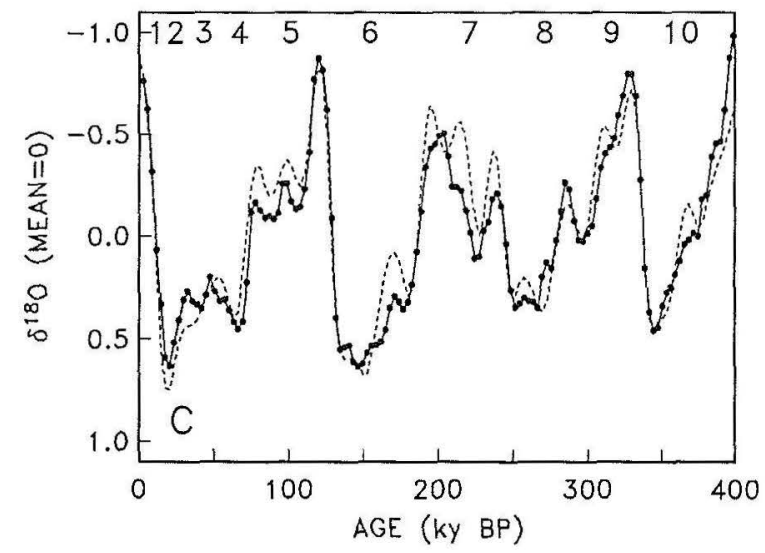

Fig. 6. Isotopic differences between cores (V19-27 minus RC13-110) plotted versus age. Differences calculated after interpolation of individual records at 3-kyr intervals and Gaussian smoothing with 6-kyr half width. (a) For $\Delta \delta 180$, more negative values indicate deep waters colder and/or more enriched in $\delta 18 \mathrm{O}$ than intermediate water. (b) For $\Delta \delta^{13} \mathrm{C}$, more positive values indicate lower nutrients (higher $\delta 13 \mathrm{C}$ ) in intermediate waters relative to deep waters. Note the rhythmic variations in the $\delta 13 \mathrm{C}$ difference. (c) Average $\delta 18 \mathrm{O}$ record from V19-27 and RC13-110, smoothed as above and mean $=0$ (solid line and dots). For comparison, the SPECMAP 8180 "long" time scale [Imbrie et al., 1984] is plotted (dashed). 
is similar to that in RC13-110, $0.13 \pm 0.04 \%$ ( $(n=3)$. Water column data from the central Pacific would predict slightly lower values for the $\delta^{13} \mathrm{C}$ value in $\sum \mathrm{CO}_{2}$ at both core sites $(0.0 \pm 0.1 \%$ [Kroopnick, $1974,1985])$. Most studies of core-top benthic foraminifera consider the ${ }^{13} \mathrm{C}$ value of Cibicides to approximate that of $\Sigma \mathrm{CO}_{2}$ in the ambient water [Woodruff et al., 1980; Vincent et al., 1981; Belanger et al., 1981; Graham et al., 1981]. Duplessy et al. [1984] suggest that Cibicides $\delta^{13} \mathrm{C}$ values may be $\sim 0.07 \pm 0.04 \%$ o higher than $\Sigma \mathrm{CO}_{2}$ values. Our data are consistent with that assertion. Because the water column data are not from locations near the core sites and because the GEOSECS Pacific data required empirical corrections after analysis [Kroopnick, 1985], we can not make a more rigorous comparison between the water column and the foraminiferal data.

In core V19-27 there is a significant shift of $\delta{ }^{13} \mathrm{C}$ values near the core top, from a mean of $0.40 \pm 0.06$ $(\mathrm{n}=6)$ from 20 to $40 \mathrm{~cm}$ depth, to $0.19 \pm 0.18(\mathrm{n}=5)$ from 0 to $20 \mathrm{~cm}$ (Figure $2 \mathrm{~b}$ ). Two of the analyses (from 5 and $12 \mathrm{~cm}$ ) are from a 26-cm-long trigger core. Agreement between the trigger core and piston core $\delta 18 \mathrm{O}$ data indicates that contamination of the piston core top with glacial-age material did not occur. It remains possible that some material may be missing from the top of the piston core. We did not attempt to correct for this, but merged the data from the piston and trigger cores at their measured depths. Thus, potential depth offsets between the trigger core and piston core, rather than analytical noise, probably account for the large apparent error $( \pm 0.18 \%)$ in the $\delta^{13} \mathrm{C}$ data from the top $20 \mathrm{~cm}$ of V19-27. Sedimentation rates in the Holocene section of this core are high, near $9 \mathrm{~cm} / \mathrm{kyr}$, making this a good site to see variations on the scale of a few thousand years. Additional analyses in other cores will be required to confirm this shift. If real, it may reflect a major water mass change within the last few thousand years, with expansion of low $-\delta 13 \mathrm{C}$ deep water in the North Pacific at the expense of high$\delta 13 \mathrm{C}$ intermediate water in the South Pacific (Figure 1). It implies that the modern watermass distributions in this area do not reflect the mean state of the ocean within the Holocene interglacial period.

\section{Rhythmic Patterns}

The carbon-isotopic differences $\left(\Delta \delta^{13} C\right)$ between the sites (Figure $6 \mathrm{~b}$ ) change in much more systematic ways with respect to $\delta 180$ than did the oxygenisotopic differences. The long-term variations in the $\delta 13 \mathrm{C}$ offset between Pacific deep and intermediate water sites are visibly rhythmic. Variations in the vertical $\delta^{13} \mathrm{C}$ gradients are small, however, on average $0.1 \%$ higher during glacial than during interglacial stages. Extreme shifts average $0.3 \%$ (ranging from 0.2 to $0.5 \%$ ).

To define the frequencies of variations in $\delta^{13} \mathrm{C}$ gradients, we apply spectral analysis to the $\delta{ }^{13} \mathrm{C}$ difference record over the last $\sim 370$ kyr [Jenkins and Watts, 1968]. The power spectrum (Figure 7a) demonstrates that the dominant rhythms are in the $1 / 100 \mathrm{kyr}^{-1}$ and $1 / 41 \mathrm{kyr}^{-1}$ frequency bands. Both of these rhythms are significantly coherent $(p \leq 0.10)$ with the oxygen-isotope (roughly ice volume) signal (Figure $7 \mathrm{~b}$ ) and with orbital eccentricity and
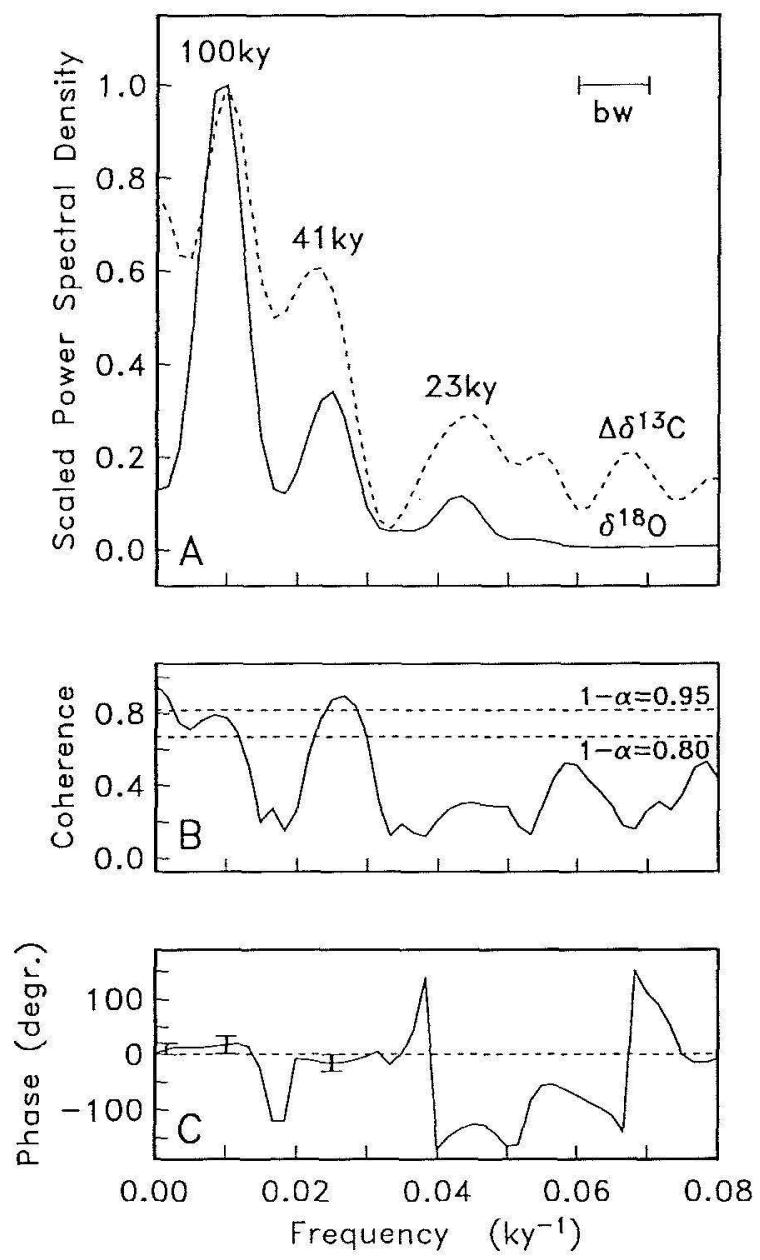

Fig. 7. (a) Power spectra (linear scale, adjusted so the peak power of the $1 / 100 \mathrm{kyr}^{-1}$ frequency $=1.0$ ) demonstrate the dominance of the $1 / 100$ and $1 / 41$ $\mathrm{kyr}^{-1}$ frequency bands in the intermediate to deepwater $\delta^{13} \mathrm{C}$ difference $\left(\Delta \delta^{13} \mathrm{C}\right.$ dashed line). For comparison, power spectrum of $\delta 18 \mathrm{O}$ is given (solid line). Bandwidth $=0.01 \mathrm{kyr}^{-1}$. (b) Significant coherency $(1-\alpha>0.80)$ occurs between the intermediate-to-deep water $\Delta \delta 13 \mathrm{C}$ and $\delta 18 \mathrm{O}$ only in the $1 / 100 \mathrm{kyr}^{-1}$ and $1 / 41 \mathrm{kyr}^{-1}$ frequency bands. (c) Phase spectrum shows that high $\delta 13 \mathrm{C}$ gradients between intermediate and deep waters co-occur (with approximately zero phase shift) with glacial maxima in the $1 / 41$ and $1 / 100 \mathrm{kyr}^{-1}$ frequency bands $(80 \%$ confidence bars for phase angle are given where significant coherency exists). 
obliquity. Amplitude, coherency, and phase of the isotope and the isotope-difference signals versus the "ETP" (eccentricity, tilt, precession) orbital index (following methods of Imbrie et al. [1989]) are given in Table 2. The phase of the intermediate-to-deep water $\Delta \delta^{13} \mathrm{C}$ difference signal is similar to that of $\delta 18 \mathrm{O}$ in the bands $1 / 100$ and $1 / 41 \mathrm{kyr}^{-1}$ frequency bands (i.e., high $\delta 13 \mathrm{C}$ gradients occur near glacial maxima). The $\Delta \delta 13 \mathrm{C}$ changes lead $\delta 18 \mathrm{O}$ slightly in the $1 / 100 \mathrm{kyr}^{-1}$ band and lag $\delta 180$ slightly in the $1 / 41$ $\mathrm{kyr}^{-1}$ band, but these offsets are marginally significant with the present resolution (Figure 7c). We infer that higher $\delta^{13} \mathrm{C}$ offsets between intermediate and deep waters, even though of small amplitude in the Pacific, are approximately linked to glacial maxima at these frequencies.

Variability of the carbon isotope differences between RC13-110 and V19-27 in the 1/19-1/23 kyr-1 frequency bands is small, and it is not significantly coherent with the oxygen isotopes (Figure $7 \mathrm{~b}$ ) or with orbital precession (Table 2). This may reflect a true lack of coherency with precession, or it may be an artifact of low signal amplitudes relative to noise in the $1 / 23$ and $1 / 19$ kyr-1 $^{-1}$ frequency bands. The question of precision is addressed in more detail below. In any case, the conclusion stands that variance in the precession band is smaller than in those of the other "Milankovitch" bands.

To test whether the variations in the carbon isotope differences between the sites are an artifact of improper correlations between cores, we repeated the analysis after correlating the $\delta 13 \mathrm{C}$ (rather than $\delta 18 \mathrm{O}$ ) signals between cores. By adjusting the time scale in V19-27, we minimized variations in the ${ }^{13} \mathrm{C}$ offsets between the sites. The result is essentially identical to that described above. The maximum concentration of variance in the $\delta 13 \mathrm{C}$ difference between sites remains in the $1 / 100 \mathrm{kyr}^{-1}$ eccentricity band and $1 / 41$ $\mathrm{kyr}^{-1}$ obliquity band. Coherence with $\delta 18 \mathrm{O}$ occurs only in these bands, and phase is consistent with highest $\delta 13 \mathrm{C}$ offsets between sites at glacial maxima The only difference in the result with this time scale is that the amplitude of variations in the $\delta 13 \mathrm{C}$ difference record is reduced by $15-20 \%$ in all frequency bands, an insignificant change. Thus, the major features of the intermediate-to-deep water $\delta^{13} \mathrm{C}$ gradients shown here are robust and unaffected by minor changes in the time scale.

\section{Variance Distribution and Signal-to-Noise Ratios}

Two questions arise when examining the importance of low amplitude signals in individual frequency bands: (1) What is the equivalent amplitude of the signal contributing to an individual spectral peak? (2) Given the analytical precision of the data, how well can we detect low amplitude, high frequency components? To address these questions, Table 2 expresses the signals in the Milankovitch bands in terms of amplitudes, and Table 3 presents calculations of signal-to-noise ratios in these bands.

TABLE 2. Amplitude (a), Coherency (k), and Phase $(\phi)$, Versus ETP (Eccentricity + Tilt + Precession) in the Milankovitch Bands, With Bandwidth $=0.01 \mathrm{kyr}^{-1}$

\begin{tabular}{|c|c|c|c|c|c|c|c|c|c|c|}
\hline \multirow[b]{2}{*}{ Core } & \multirow[b]{2}{*}{ idicator } & \multicolumn{3}{|c|}{$100 \mathrm{kyr}$} & \multicolumn{3}{|c|}{$41 \mathrm{kyr}$} & \multicolumn{3}{|c|}{$23+19 \mathrm{kyr}$} \\
\hline & & a & $\mathrm{k}$ & $\phi$ & $\bar{a}$ & $\mathrm{k}$ & $\phi$ & a & $\mathrm{k}$ & $\bar{\phi}$ \\
\hline V19-27 & $-\delta^{18} \mathrm{O}$ & 0.35 & 0.88 & $10 \pm 10$ & 0.21 & 0.88 & $81 \pm 10$ & 0.15 & 0.75 & $87 \pm 15$ \\
\hline $\mathrm{RC} 13-110$ & $-\delta^{180}$ & 0.42 & 0.90 & $-1 \pm 9$ & 0.24 & 0.90 & $86 \pm 9$ & 0.16 & 0.87 & $76 \pm 10$ \\
\hline $\mathrm{V} 19-30^{\mathrm{a}}$ & $-\delta^{18} \mathrm{O}$ & 0.35 & 0.92 & $10 \pm 8$ & 0.24 & 0.91 & $80 \pm 8$ & 0.17 & 0.89 & $98 \pm 9$ \\
\hline V19-27 & $\delta^{13} \mathrm{C}$ & 0.13 & $(0.62)$ & $24 \pm 20$ & 0.08 & 0.84 & $79 \pm 12$ & 0.04 & 0.77 & $71 \pm 14$ \\
\hline $\mathrm{RC} 13-110$ & $\delta^{13} \mathrm{C}$ & 0.18 & 0.75 & $6 \pm 15$ & 0.12 & 0.94 & $91 \pm 7$ & 0.06 & $(0.55)$ & $85 \pm 23$ \\
\hline $\mathrm{V} 19-30^{\mathrm{a}}$ & $\delta^{13} \mathrm{C}$ & 0.24 & 0.83 & $-7 \pm 12$ & 0.12 & 0.85 & $81 \pm 11$ & 0.06 & 0.81 & $106 \pm 13$ \\
\hline Deep-intermediate ${ }^{b}$ & $\Delta \delta^{13} \mathrm{C}$ & 0.09 & 0.72 & $-8 \pm 16$ & 0.07 & 0.75 & $94 \pm 15$ & 0.06 & $(0.26)$ & $-165 \pm 39$ \\
\hline Deep-surface ${ }^{c}$ & $\Delta \delta^{13} \mathrm{C}$ & 0.11 & 0.92 & $29 \pm 8$ & 0.06 & 0.90 & $62 \pm 9$ & 0.04 & 0.77 & $51 \pm 14$ \\
\hline
\end{tabular}

The 0.20 significance level $(1-\alpha=0.80)$ for $k=0.67$. Values with insignificant coherencies are in parentheses. Confidence limits on $\phi$ are at the $80 \%$ level.

aV19-30 Uvigerina sp.[Shackleton and Pisias, 1985].

bRC13-110 - V19-27 [this paper].

cV19-30 Uvigerina sp. - N. dutertrei [Shackleton and Pisias, 1985]. 
Mix et al.: Carbon 13 in Pacific Deep and Intermediate Waters, 0-370 ka

TABLE 3. Signal/Noise Ratios in the Milankovitch Frequency Bands

\begin{tabular}{|c|c|c|c|c|}
\hline Core & Indicator & $100 \mathrm{kyr}$ & $41 \mathrm{kyr}$ & $23+19 \mathrm{kyr}$ \\
\hline $\begin{array}{l}\text { V19-27 } \\
\text { RC13-110 } \\
\text { V19-30a }\end{array}$ & $\begin{array}{l}-\delta 18 \mathrm{O} \\
-\delta 18 \mathrm{O} \\
-\delta 18 \mathrm{O}\end{array}$ & $\begin{array}{l}69 \\
74 \\
69\end{array}$ & $\begin{array}{l}25 \\
23 \\
31\end{array}$ & $\begin{array}{r}8.6 \\
6.5 \\
11\end{array}$ \\
\hline $\begin{array}{l}\text { V19-27 } \\
\text { RC13-110 } \\
\text { V19-30a }\end{array}$ & $\begin{array}{l}\delta^{13} \mathrm{C} \\
\delta^{13} \mathrm{C} \\
\delta^{13} \mathrm{C}\end{array}$ & $\begin{array}{l}30 \\
42 \\
94\end{array}$ & $\begin{array}{l}10 \\
23 \\
23\end{array}$ & $\begin{array}{l}2.5 \\
3.3 \\
5.0\end{array}$ \\
\hline $\begin{array}{l}\text { Deep-intermediate } \\
\text { Deep-surface }\end{array}$ & $\begin{array}{l}\Delta \delta^{13} \mathrm{C} \\
\Delta \delta^{13} \mathrm{C}\end{array}$ & $\begin{array}{r}6.4 \\
11\end{array}$ & $\begin{array}{l}3.6 \\
3.3\end{array}$ & $\begin{array}{l}2.9 \\
1.7\end{array}$ \\
\hline F statistic ${ }^{d}$ & $F_{(.90)}$ & 2.8 & 2.8 & 2.2 \\
\hline
\end{tabular}

aV19-30 Uvigerina sp. [Shackleton and Pisias, 1985].

bRC13-110 - V19-27 (this paper).

cV19-30 Uvigerina sp. - N. dutertrei [Shackleton and Pisias, 1985].

$\mathrm{dF}$ statistic for ratio of variances. For the $1 / 100$ and $1 / 41 \mathrm{kyr}^{-1}$ frequencies, degrees of freedom are 7,7 (one spectral bandwidth). For the $1 / 23 \mathrm{kyr}^{-1}$ frequency band, degrees of freedom are 12,12

(1.7 spectral bandwidths). Signal/Noise ratios greater than $\mathrm{F}_{(.90)}$ are significant $(p \leq .010)$.

These calculations are based on the fact that the integral of the power spectral density function over the frequency interval of zero to the nyquist frequency $(0.5$ cycles/sample interval); i.e., the area under the spectral density curve, equals the total variance of the original time series. To determine the variance contained within a frequency band, we calculate the fraction of the total area under the spectral density curve which is contained in the selected frequency range, and multiply this fraction by the total variance. For the $1 / 100$ and $1 / 41 \mathrm{kyr}^{-1}$ spectral peaks we integrate the spectral density function over frequency bands centered at the $1 / 100$ and $1 / 41 \mathrm{kyr}^{-1}$ peaks, respectively. The width of each band is set to one bandwidth of the spectral calculation (here $0.01 \mathrm{kyr}^{-1}$ ). This is because all spectral estimates within a bandwidth are correlated. The bandwidth represents the resolution of the frequency spectrum. For the precessional band we integrate over a range that includes both the $1 / 23$ and $1 / 19 \mathrm{kyr}^{-1}$ frequencies, equal to 1.7 bandwidths.

To estimate the amplitude in each frequency band we use the equation:

$$
\mathrm{a}_{\mathrm{f}}=\sqrt{2 \mathrm{~s}_{\mathrm{f}}^{2}}
$$

where $a_{f}$ is amplitude and $s_{f}^{2}$ is the variance within a frequency band. This assumes that the variance contained in a given frequency band is produced by a pure sine wave. This calculation gives the amplitudes listed in Table 2, in the original units of measurement (\%o). The full range of values in the time domain, represented by each frequency band, can be estimated as approximately 2 times this amplitude.
To address the second question concerning the relationship between the variances and derived amplitudes, and analytical error, in Table 3 we calculate a signal-to-noise ratio within each frequency band for each of the time series of Table 2. The noise level within the spectral density function is estimated using, again, the property that the area under the curve of pure noise in the frequency range from zero to the nyquist frequency is equal to the total variance of noise. The observed analytical precision of 48 replicate analyses of $C$. wuellerstorfi in core V19-27 yields variance estimates of 0.023 $(\% o)^{2}$ for $\delta 18 \mathrm{O}$ and $0.007(\% \circ)^{2}$ for $\delta 13 \mathrm{C}$ in individual samples (i.e., standard deviations of 0.15 and $0.08 \%$ respectively). We assume that this level of analytical noise applies randomly to all analyses and thus would apply equally to all frequency bands. Smoothing of the records when interpolating to constant time intervals for the spectral analysis reduces the total noise level slightly in all cores. The existence of replicates in V19-27 and a small time sampling interval in V19-30 reduces random errors further in these cores, and we include this effect in our calculations. The signal-to-noise ratio (Table 3) is calculated from the total variance (signal plus noise) observed within each frequency band divided by the variance expected solely from random analytical errors. Large values of $\mathrm{S} / \mathrm{N}$ increase our confidence in the results, and low values suggest caution. A value of 1.0 would indicate no signal over the expected noise level, while a value of 2.0 would indicate equal amounts of signal and noise.

The results in Table 3 indicate that signal/noise ratios are greater than one in all cases. The lowest values $(<3)$ occur in the precession band $(1 / 23+$ 
$1 / 19 \mathrm{kyr}^{-1}$ ) for the $\delta^{13} \mathrm{C}$ record from $\mathrm{V} 19-27$ and both the intermediate-to-deep water and surface- todeep water $\delta 13 \mathrm{C}$ difference estimates. All values in Table 3, with the exception of deep-surface $\Delta \delta{ }^{13} \mathrm{C}$ record from Shackleton and Pisias [1985], have signal-to-noise ratios higher than $\mathrm{F}_{(.90)}$; i.e., they are significantly different from 1.0 . These results indicate that the spectral analysis of these data is on safe ground in the $1 / 100$ and $1 / 41 \mathrm{kyr}^{-1}$ bands but that inferences from carbon isotope data in the precession band should be treated with caution.

\section{CONCLUSIONS}

The primary result here, from analysis of the first long time series of benthic foraminiferal stable isotope data from intermediate depths in the Pacific Ocean, is that variations of intermediate-to-deep water $\delta{ }^{13} \mathrm{C}$ gradients in the Pacific are very small. This assumes that the $\delta^{13} \mathrm{C}$ record from V19-27 is representative of the whole intermediate-depth Pacific. Our results are quite different from those in the Atlantic, where large vertical $\delta{ }^{13} \mathrm{C}$ gradients during glacial maxima (the Boyle effect) imply strong nutrient depletion of intermediate waters. Our results are consistent with the existence of a very weak Boyle effect in the Pacific, with a glacial-interglacial contrast of $0.1 \%$ (averaged over whole stages), to $0.3 \%$ o (average peak-to-peak differences between glacial and interglacial stages). This is much smaller than Boyle's [1988] box model prediction of $\sim 0.8 \%$ o for the global average increase in the intermediate-todeep $\delta{ }^{13} \mathrm{C}$ gradient, which in the model could account for up to half of the observed atmospheric $\mathrm{pCO}_{2}$ changes. Given the larger changes in ${ }^{113} \mathrm{C}$ gradients in parts of the Indian and Atlantic oceans, the mean global $\delta 13 \mathrm{C}$ gradient between intermediate and deep waters at glacial maxima may have been larger than we calculate for the Pacific. The large volume of the Pacific, however, makes its variations relatively important for the global average. Thus, it is unlikely that the global average amplitude of the Boyle effect approached the values measured in the Atlantic. If so, the global nutrient transfer effect is probably smaller than that required by the box models to explain even half of the ice core $\mathrm{CO}_{2}$ record [Boyle, 1988]. The possibility remains that the box model under-estimates sensitivity of the $\mathrm{CO}_{2}$ system to this effect. In any case, the addition of Pacific data adds another constraint that must be considered.

If we assume for now that the Pacific intermediate-to-deep water $\delta^{13} \mathrm{C}$ gradient reflects Boyle's mechanism of stripping nutrients and carbon from intermediate waters, it would appear that it operates most effectively in the $1 / 100$ and $1 / 41 \mathrm{kyr}^{-1}$ frequency bands. In contrast, the Vostok ice core data [Barnola et al., 1987] has relatively little variance in the $1 / 41 \mathrm{kyr}^{-1}$ band. Although the time scales for comparing ice-core $\mathrm{CO}_{2}$ and marine sedimentary records are not yet final [Petit et al., 1990], it appears that $\mathrm{CO}_{2}$ has most of its variance near the $1 / 100 \mathrm{kyr}^{-1}$ band and near the $1 / 23 \mathrm{kyr}^{-1}$ band, which may be coherent with orbital eccentricity and precession [Lyle and Pisias, 1990]. The variations in intermediate-to-deep water $\Delta \delta{ }^{13} \mathrm{C}$ signal calculated here lag those of the surface-to-deep water $\triangle \delta{ }^{13} \mathrm{C}$ signal of Shackleton and Pisias [1985] (which mimic the ice core $\mathrm{CO}_{2}$ record), by $20-30^{\circ}$ in the dominant $1 / 100$ and $1 / 41 \mathrm{kyr}^{-1}$ frequency bands (Table 2). Clearly, more work must be done to extract the global-scale signals from local signals in the deep ocean. For now, however, we believe it is safe to conclude that mismatches between the ice core $\mathrm{CO}_{2}$ and the various $\delta 13 \mathrm{C}$ records require that more than one mechanism must exist to account for variations in the distribution of carbon in the ocean and atmosphere.

With the data presently available, we can not exclude the possibility of local water mass changes in the Pacific masking the large-scale effect of nutrient transfer to deep water at glacial maxima. Significant horizontal gradients in water column $\delta 1^{13} \mathrm{C}$ exist in the mid-depth Pacific. At present, high- $\delta 13 \mathrm{C}(0.1$ $0.5 \%$ ) intermediate waters of Antarctic origin dominate the southern hemisphere, while low- $\delta{ }^{13} \mathrm{C}$ (0 to $-0.3 \%$ ) waters dominate mid-depths of the North Pacific [Kroopnick, 1985]. Core V19-27 lies in the mixing zone between these water masses (Figure 1). With data from just this one intermediate-water site in the Pacific, a scenario is possible whereby no Boyle effect occurs in the Pacific and northward migration of the gradient between the two present water masses at the glacial maximum drives the $\Delta \delta{ }^{13} \mathrm{C}$ difference signal. Equally possible, there could be a stronger Boyle effect in the Pacific at the glacial maximum, which was masked at the site of V19-27 by southward movements of the watermass gradients. The relatively large $\delta{ }^{13} \mathrm{C}$ changes near the core top in V19-27, if real, suggest to us that local water mass changes may be very important in this region. Better constraints on the intermediate water carbon isotope budget and circulation changes will require analyzing the spatial patterns of $\delta 13 \mathrm{C}$ at several intermediatewater sites in the Pacific and throughout the world oceans.

Acknowledgments. This work was funded by the National Science Foundation and was initiated by the NSF Research Experience for Undergraduates program. The authors thank D. Oppo and an anonymous reviewer for helpful comments and $\mathrm{E}$. Boyle, T. Hagelberg, and J. Imbrie for discussions. Assistance from A. Morey on graphics is appreciated. For curation of cores, we acknowledge the National Science Foundation and the Office of Naval Research. 


\section{REFERENCES}

Barnola, J.M., D. Raynaud, Y.S. Korotkevich, and C. Lorius, Vostok ice core provides 160,000 year record of atmospheric $\mathrm{CO}_{2}$, Nature, $329,408-$ 414, 1987.

Belanger, P., W.B. Curry, and R.K. Matthews, Core-top evaluation of benthic foraminiferal isotopic ratios for paleo-oceanographic interpretations, $\underline{\text { Palaeogeogr. Palaeoclimatol. }}$ Palaeoecol, 33, 205-220, 1981.

Boyle, E.A., Deep ocean circulation, preformed nutrients, and atmospheric carbon dioxide: Theories and evidence from oceanic sediments, in Mesozoic and Cenozoic Oceans. Geodyn. Ser., vol 15 , edited by K. Hsu, pp. 49-60, AGU, Washington, D.C., 1986.

Boyle, E.A., The role of vertical chemical fractionation in controlling late Quaternary atmospheric carbon dioxide, J, Geophys, Res., 93. 15,701-15,714, 1988.

Boyle, E.A., and L.D. Keigwin, Comparison of Atlantic and Pacific paleochemical records for the last 215,000 years: Changes in deep ocean circulation and chemical inventories, Earth Planet. Sci. Lett. 76, 135-150, 1985/1986.

Boyle, E.A., and L.D. Keigwin, North Atlantic thermohaline circulation during the last 20,000 years linked to high-latitude surface temperature, Nature, 330, 35-40, 1987.

Broecker, W.S., Glacial to interglacial changes in ocean chemistry, Prog. Oceanogr. 11, 151-197, 1982.

Broecker W.S., T.-H. Peng, and R. Engh, Modeling the carbon system, Radiocarbon, 22, 565-598, 1980.

Cofer-Shabica, N., and L. Peterson, Caribbean carbon isotope record for the last 300,000 years, Geol. Soc. Am. Abstr. Programs, 18, 567, 1986.

Duplessy, J.-C., and N.J. Shackleton, Response of global deep-water circulation to the Earth's climatic change $135,000-107,000$ years ago, Nature, 316, 500-507, 1985.

Duplessy, J.-C., N.J. Shackleton, R.G. Fairbanks, L. Labeyrie, D. Oppo, and N. Kallel, Deep-water source variations during the last climatic cycle and their impact on the global deep-water circulation, Paleoceanography, 3, 343-360, 1988.

Duplessy, J.-C., N.J. Shackleton, R.K. Matthews, W. Prell, W.F. Ruddiman, M. Caralp, and C.H. Hendy, ${ }^{13} \mathrm{C}$ record of benthic foraminifera in the last interglacial ocean: Implications for the carbon cycle and the global deep water circulation, Quat. Res. 21, 225-243, 1984.

Graham, D.W., B.H. Corliss, M.L. Bender, and L.D. Keigwin, Carbon and oxygen isotopic disequilibria of recent deep-sea benthic foraminifera, Mar, Micropaleontol., 6 , 483-497, 1981.
Imbrie, J., J.D. Hays, D.G. Martinson, A. McIntyre, A.C. Mix, J.J. Morley, N.G. Pisias, W.L. Prell, and N.J. Shackleton, The orbital theory of Pleistocene climate: support from a revised chronology of the marine $\delta 18 \mathrm{O}$ record, in Milankovitch and Climate, vol. 1., edited by A. Berger et al., pp. 269-305, D. Reidel, Hingham, Mass., 1984.

Imbrie, J., A. McIntyre, and A.C. Mix, Oceanic response to orbital forcing in the late Quaternary: Observational and experimental strategies, in Climate and Geo-sciences, edited by A. Berger, S. Schneider, and J.-C. Duplessy, pp. 121-164, Kluwer Academic, Boston, Mass., 1989.

Jenkins, G.M., and D.G. Watts, Spectral Analysis and Its Applications, 525 pp., Holden-Day, San Francisco, Calif., 1968.

Kallel, N., L.D. Labeyrie, A. Juillet-Leclerc, and J.C. Duplessy, A deep hydrological front between intermediate and deep-water masses in the glacial Indian Ocean, Nature, 333, 651-655, 1988.

Keir, R.S., On the late Pleistocene ocean geochemistry and circulation, Paleoceanography, 3, 413-446, 1988.

Knox, F., and M. McElroy, Changes in atmospheric CO2: Influence of the marine biota at high latitude, J. Geophys. Res., 89, 4629-4637, 1985.

Kroopnick, P., The dissolved $\mathrm{O}_{2}-\mathrm{CO}_{2}-13 \mathrm{C}$ system in the eastern equatorial Pacific, Deep Sea Res., 21. 211-227, 1974.

Kroopnick, P., The distribution of ${ }^{13} \mathrm{C}$ of $\sum \mathrm{CO}_{2}$ in the world oceans, Deep Sea Res., 32, 57-84, 1985.

Labeyrie L.D., and J.-C. Duplessy, Changes in the oceanic $\mathrm{C}^{13} / \mathrm{C}^{12}$ ratio during the last 140,000 years: High latitude surface water records, Palaeogeogr. Palaeoclimatol. Palaeoecol., 50. 217-240, 1985.

Levitus, Climatological Atlas of the World Ocean, NOAA Prof, Pap. 13 pp. 1-171, U.S. Dep. Commer., Washington, D.C., 1982.

Lyle, M., and N.G. Pisias, Ocean circulation and atmospheric $\mathrm{CO}_{2}$ changes: Coupled use of models and paleoceanographic data, Paleoceanography, 5 $15-41,1990$.

Martinson, D., W. Menke, and P. Stoffa, An inverse approach to signal correlation, J.Geophys. Res., 87. 4807-4818, 1982.

Oppo, D., and R.G. Fairbanks, Variability in the deep and intermediate water circulation of the Atlantic Ocean during the past 25,000 years: Northern hemisphere modulation of the southern ocean, Earth Planet. Sci. Lett., 86, 1-15, 1987.

Oppo, D., and R.G. Fairbanks, Atlantic Ocean thermohaline circulation of the last 150,000 years: Relationship to climate and atmospheric $\mathrm{CO}_{2}$, Paleoceanography, 5, 277-288, 1990.

Pedersen, T.F., M. Pickering, J.S. Vogel, J.N. Southon, and D.E. Nelson, The response of 
benthic foraminifera to productivity cycles in the eastern equatorial Pacific: Faunal and geochemical constraints on glacial bottom-water oxygen levels, Paleoceanography, 3, 157-168, 1988.

Petit, J.R., L. Mounier, J. Jouzel, Y.S.

Korotkevich, V.I. Kotlyokov, and C. Lorius, Palaeoclimatological and chronological implications of the Vostok core dust record, Nature, 343, 56-58, 1990.

Sarmiento, J.L., and J.R. Toggweiler, A new model for the role of the oceans in determining atmospheric $\mathrm{pCO}_{2}$, Nature, 308, 621-624, 1985.

Shackleton, N.J., Attainment of isotopic equilibrium between ocean water and the benthonic foraminiferal genus Uvigerina; Isotopic changes in the ocean during the last glacial, Colloques Int. Cent. Natn, Rech, Scient., 219, 203-209, 1974.

Shackleton, N.J., and N. Opdyke, Oxygen isotopic and paleomagnetic stratigraphy of equatorial Pacific core V28-238: Oxygen isotope temperatures and ice volumes on a $10^{5}$ and $10^{6}$ year scale, Quat, Res., 3. 39-55, 1973.

Shackleton, N.J., and N.G. Pisias, Atmospheric carbon dioxide, orbital forcing, and climate, in The Carbon Cycle and Atmospheric $\mathrm{CO}_{2}$; Natural Variations Archean to Present, Geophys. Monogr. Ser., 32, pp. 313-318, edited by E.T. Sundquist and W.S. Broecker, AGU, Washington, D.C., 1985.

Siegenthaler, U., and T. Wenk, Rapid atmospheric $\mathrm{CO} 2$ variations and ocean circulation, Nature, 308, 624-625, 1984.

Suess, E., Particulate organic carbon flux in the oceans - Surface productivity and oxygen utilization, Nature, 288, 260-263, 1980.

Vincent, E., J.S. Killingley, and W.H. Berger, Stable isotope composition of benthic foraminifera from the equatorial Pacific, Nature, 289, 639-643, 1981.

Woodruff, F., S.M. Savin, and R. Douglas, Biological fractionation of oxygen and carbon isotopes by recent benthic foraminifera, $\mathrm{Mar}$. Micropaleontol., 5, 3-11, 1980.

Zahn, R, and T.F. Pedersen, Late Pleistocene evolution of surface and mid-depth hydrography at the Oman Margin: Planktonic and benthic isotope records at ODP Site 724, in Proceedings of the Ocean Drilling Program. Part B: Scientific Results, Leg 117, edited by W.L. Prell, and N. Niitsuma, in press, 1990.

Zahn, R., K. Winn, and M. Sarnthein, Benthic foraminiferal $\delta^{13} \mathrm{C}$ and accumulation rates of organic carbon: Uvigerina peregrina group and Cibicidoides wuellerstorfi, Paleoceanography, 1 . 27-42, 1986.

Zahn, R., M. Sarnthein, and H. Erlenkeuser, Benthic isotope evidence for changes of the Mediterranean outflow during the late Quaternary, Paleoceanography, 2. 543-560, 1987.

C. Lopez, A.C. Mix, N.G. Pisias, and W. Rugh, College of Oceanography, Oregon State University, Corvallis, OR 97331.

K. Nelson, Physics Department, University of California, Berkeley, CA 94720.

R. Zahn, GEOMAR Wischhofstrasse 1-3, D-2300 Kiel 14, Federal Republic of Germany.

(Received May 3, 1990; revised October 15, 1990; accepted October 16, 1990.) 